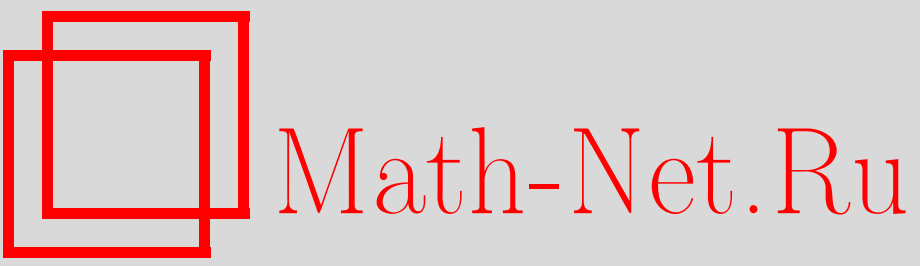

Д.В. Глазков, Локальный асимптотический анализ моделей оптикоэлектронных систем с запаздыванием, Итоги науки и техн. Сер. Соврем. мат. и ее прил. Темат. обз., 2021, том 190, 57-80

DOI: https://doi.org/10.36535/0233-6723-2021-190-57-80

Использование Общероссийского математического портала Math-Net.Ru подразумевает, что вы прочитали и согласны с пользовательским соглашением

http: //www.mathnet.ru/rus/agreement

Параметры загрузки:

IP : 54.224 .60 .19

26 апреля 2023 г., $16: 33: 56$ 


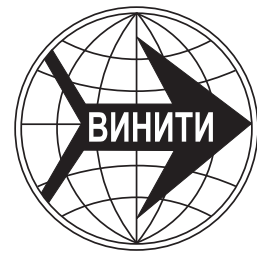

ИТОГИ НАУКИ И ТЕХНИКИ.

Современная математика и ее приложения.

Тематические обзоры.

Том 190 (2021). C. $57-80$

DOI: $10.36535 / 0233-6723-2021-190-57-80$

УДК 517.929

\title{
ЛОКАЛЬНЫЙ АСИМПТОТИЧЕСКИЙ АНАЛИЗ МОДЕЛЕЙ ОПТИКО-ЭЛЕКТРОННЫХ СИСТЕМ С ЗАПАЗДЫВАНИЕМ
}

\author{
(c) 2021 г. Д. В. ГЛАЗКОВ
}

\begin{abstract}
АннотАция. Проведено математическое исследование нескольких моделей одного класса оптико-электронных систем с запаздывающей обратной связью, которые являются обобщениями известной модели Ланга-Кобаяси полупроводникового лазера. Предлагаются новые редуцированные уравнения, которые описывают динамику исходных систем в окрестности предельных семейств периодических решений при асимптотически больших значениях одного из параметров задачи. На основе их численно-аналитического изучения делаются некоторые выводы прикладного характера.
\end{abstract}

Ключевые слова: математическая модель, дифференциальное уравнение с запаздыванием, полупроводниковый лазер, малый параметр, асимптотика, устойчивость, бифуркация.

\section{LOCAL ASYMPTOTIC ANALYSIS \\ OF MODEL OPTOELECTRONIC SYSTEMS WITH DELAY}

\author{
(C) 2021 D. V. GLAZKOV
}

\begin{abstract}
In this paper, we examine several models of optoelectronic systems with delayed feedback that are generalizations of the well-known Lang-Kobayashi model of semiconductor lasers. We propose new reduced equations that describe the dynamics of the original systems in a neighborhood of limit families of periodic solutions for asymptotically large values of a parameter of the problem. Using numerical and analytical methods, we obtain some applied conclusions.
\end{abstract}

Keywords and phrases: mathematical model, differential equation with delay, semiconductor laser, small parameter, asymptotics, stability, bifurcation.

AMS Subject Classification: 34E10, 39A10, 39A11

1. Введение. Модели, описывающие динамику оптико-электронных систем, на протяжении многих лет являются объектами повышенного внимания со стороны многочисленных исследователей (см. $[13,27,32,34])$. В первую очередь это обусловлено их непосредственным прикладным значением. Достаточно сказать, что интернет в привычном нам виде был бы невозможен без оптико-электронных коммуникаций. Однако эта причина не единственная. Как отмечено в [13], лазер принадлежит к числу систем, которые не только способны демонстрировать сложное поведение, но и более многих других пригодны для исследования общих закономерностей нелинейной динамики. Действительно, в целом ряде случаев лазеры функционируют в существенно нелинейных режимах. В наибольшей степени это замечание касается так называемой полуклассической теории лазера, которая предлагает целую иерархию нелинейных уравнений, надежно обоснованных с позиций квантовой электродинамики и количественно подтвержденных экспериментами.

Работа выполнена при поддержке Российского фонда фундаментальных исследований (проект № 18-29-10043). 
Особый класс моделей оптико-электронных систем представляют собой модели, учитывающие воздействие запаздывающей оптической обратной связи. Их сложность обусловлена тем, что этот класс задач описывается в терминах дифференциальных уравнений с запаздыванием, которые обладают бесконечным числом степеней свободы. Кроме того, динамические системы с запаздыванием демонстрируют различные типы неустойчивостей, обусловленных воздействием задержки. Например, для полупроводниковых лазеров с оптической обратной связью наблюдаются различные пути перехода к хаосу: квазипериодический (см. $[18,19])$, через каскад бифуркаций удвоения периода (см. [37]), сценарий Икеды (см. [14]), перемежаемость и кризис аттракторов (см. [29,30]).

В таких областях как хранение данных или оптические и оптоволоконные коммуникации отражения и связанные с ними сопутствующие эффекты неизбежны. Поэтому изучение влияния оптической запаздывающей обратной связи на работу лазеров разных типов представляет собой важную прикладную задачу.

Данная работа является продолжением серии статей [2-4], посвященных исследованию особенностей динамики моделей оптико-электронных систем с отклоняющимся аргументом, и обобщает результаты, полученные в [3], на случай некоторых моделей многомодового полупроводникового лазера.

2. Постановка задачи. Рассматриваемые в работе математические модели динамики многомодового полупроводникового лазера являются обобщениями известной системы уравнений Ланга-Кобаяси (см. [23]), которая описывает поведение одномодового лазера и имеет вид

$$
\left\{\begin{array}{l}
\frac{d E}{d t}=v(1+i \alpha) E Z+\gamma e^{-i \omega_{0} h} E(t-h), \\
\frac{d Z}{d t}=Q-Z-(1+Z)|E|^{2} .
\end{array}\right.
$$

Здесь $E(t)$ - комплексная амплитуда электрического поля, $Z(t)$ - инверсия носителей; $\gamma>0$ и $-\omega_{0} h-$ сила и фаза обратной связи $(\mathrm{OC}), \omega_{0}$-оптическая частота генерации в отсутствие обратной связи; $Q$ - превышение током накачки первой пороговой величины; $v$-отношение времен затухания инверсии носителей и фотонов в резонаторе; $\alpha$-коэффициент уширения линии, отвечающий за нелинейное взаимодействие между амплитудой и фазой поля; $h$ - время прохода излучения по внешнему резонатору, нормированное в единицах времени затухания инверсии.

Простейшая модель многомодового лазера, подвергающегося воздействию запаздывающей обратной связи, приведена в [35] и может быть выписана в следующем виде:

$$
\left\{\begin{array}{l}
\frac{d E_{m}}{d t}=v(1+i \alpha) E_{m} Z_{m}+\gamma_{m} e^{-i \Omega_{m} h} E_{m}(t-h), \\
\frac{d Z_{m}}{d t}=Q-Z_{m}-\left(1+Z_{m}\right) \sum_{j=1}^{n}\left|E_{j}\right|^{2},
\end{array}\right.
$$

где $n$ - число мод когерентного оптического излучения, индекс $m$ изменяется от 1 до $n, \Omega_{m}-$ оптические частоты лазерных мод без ОС, величины OC $\gamma_{m}$ в простейшем случае одинаковы и от $m$ не зависят. Остальные параметры имеют тот же смысл, что и в базовой одномодовой модели Ланга-Кобаяси.

Существует модификация этой модели (см. [8]), которая, по мнению ее автора, более корректно учитывает взаимодействие между модами:

$$
\left\{\begin{array}{l}
\frac{d E_{m}}{d t}=v(1+i \alpha)\left[\gamma_{m}\left(Z_{0}-Z_{m}\right)-1\right] E_{m}+\gamma e^{-i \Omega_{m} h} E_{m}(t-h) \\
\frac{d Z_{m}}{d t}=-\left(1+d+\sum_{j=1}^{n} \gamma_{j}\left|E_{j}\right|^{2}\right) Z_{m}+\gamma_{m}\left|E_{m}\right|^{2} Z_{0} / 2 \\
\frac{d Z_{0}}{d t}=Q-Z_{0}+\sum_{j=1}^{n}\left|E_{j}\right|^{2}\left(Z_{j}-Z_{0}\right) .
\end{array}\right.
$$


Здесь $E_{m}$ - медленно меняющиеся комплексные амплитуды полей мод, $Z_{0}$ и $Z_{m}$ - пространственно однородная компонента и амплитуда пространственной решетки плотности неравновесных носителей, $\Omega_{m}$ - оптические частоты лазерных мод без ОС, $\gamma_{m}-$ коэффициент усиления $m$-й моды относительно усиления моды, ближайшей к центру линии, $d$-безразмерный коэффициент диффузии.

Рассмотрим модификацию исходной модели (1) полупроводникового лазера, в которой добавляется еще одно уравнение, описывающее воздействие оптического фильтра (см. [17]):

$$
\left\{\begin{array}{l}
\frac{d E}{d t}=v(1+i \alpha) E Z+\gamma f \\
\frac{d f}{d t}=i \Delta f+\Lambda\left[E(t-h) e^{-i \omega_{0} h}-f\right] \\
\frac{d Z}{d t}=Q-Z-(1+Z)|E|^{2} .
\end{array}\right.
$$

Здесь $f(t)$ - комплексная амплитуда электрического поля на выходе из фильтра, $\Lambda$ - ширина спектра, $\Delta$-расстройка между частотой излучения уединенного лазера и несущей частотой фильтра.

Описанный в [17] механизм работы оптического фильтра может быть использован и для многомодовых полупроводниковых лазеров. В случае системы (2) соответствующая модель будет описываться следующими уравнениями:

$$
\left\{\begin{array}{l}
\frac{d E_{m}}{d t}=v(1+i \alpha) E_{m} Z_{m}+\gamma f_{m} \\
\frac{d f_{m}}{d t}=i \Delta_{m} f_{m}+\Lambda\left[E_{m}(t-h) e^{-i \Omega_{m} h}-f_{m}\right] \\
\frac{d Z_{m}}{d t}=Q-Z_{m}-\left(1+Z_{m}\right) \sum_{j=1}^{n}\left|E_{j}\right|^{2}
\end{array}\right.
$$

Система (3) модифицируется следующим образом:

$$
\left\{\begin{array}{l}
\frac{d E_{m}}{d t}=v(1+i \alpha)\left[\gamma_{m}\left(Z_{0}-Z_{m}\right)-1\right] E_{m}+\gamma f_{m} \\
\frac{d f_{m}}{d t}=i \Delta_{m} f_{m}+\Lambda\left[E_{m}(t-h) e^{-i \Omega_{m} h}-f_{m}\right] \\
\frac{d Z_{m}}{d t}=-\left(1+d+\sum_{j=1}^{n} \gamma_{j}\left|E_{j}\right|^{2}\right) Z_{m}+\gamma_{m}\left|E_{m}\right|^{2} Z_{0} / 2 \\
\frac{d Z_{0}}{d t}=Q-Z_{0}+\sum_{j=1}^{n}\left|E_{j}\right|^{2}\left(Z_{j}-Z_{0}\right) .
\end{array}\right.
$$

В (5), (6) величины $f_{m}(t)$ - комплексные амплитуды электрических полей мод на выходе из фильтра, $\Delta_{m}$ - расстройки между оптическими частотами $\Omega_{m}$ лазерных мод без ОС и несущей частотой фильтра.

Ставится задача математического исследования локальной динамики представленных систем дифференциальных уравнений в предположении, что значения параметров $Q$ асимптотически велики.

Наличие в системе большого параметра приводит к необходимости рассматривать сингулярно возмущенную задачу. Для изучения происходящих в системе бифуркаций используется метод построения специальных нормализованных уравнений для медленных амплитуд, которые описывают поведение определенных решений исходной задачи. Важной особенностью этих уравнений является то, что от больших (малых) параметров они не зависят.

3. Простейшие свойства одномодовых моделей. В этом разделе вкратце приводятся некоторые известные свойства рассматриваемых моделей. 
Если выполнить в (1) замену $E(t)=R(t) \cdot e^{i \theta(t)}$, то можно перейти к уравнениям в более удобной для расчетов форме относительно вещественной амплитуды и фазы поля:

$$
\left\{\begin{array}{l}
\frac{d R}{d t}=v R Z+\gamma \cos \left(\omega_{0} h+\theta-\theta(t-h)\right) R(t-h), \\
\frac{d \theta}{d t}=v \alpha Z-\gamma \sin \left(\omega_{0} h+\theta-\theta(t-h)\right) \frac{R(t-h)}{R} \\
\frac{d Z}{d t}=Q-Z-(1+Z) R^{2} .
\end{array}\right.
$$

Простейшие решения системы уравнений Ланга-Кобаяси (1) определяются из условий

$$
E(t)=R_{k} \cdot e^{i\left(\omega_{k}-\omega_{0}\right) t}, \quad Z(t)=Z_{k},
$$

где $R_{k}, \omega_{k}, Z_{k}$ - константы. Такие периодические решения называются модами внешнего резонатора (external cavity modes) в [28] или модами составного резонатора (compound-cavity modes) в [34]. В обозначениях системы (7) они имеют вид

$$
R(t)=R_{k}, \quad \theta(t)=\left(\omega_{k}-\omega_{0}\right) t, \quad Z(t)=Z_{k} .
$$

Подставляя их в систему $(7)$, вводя обозначение $\eta_{k}=\omega_{k} h$ и исключая величину $R_{k}$ при условии $R_{k} \neq 0$, получим следующие соотношения для определения мод внешнего резонатора:

$$
\begin{gathered}
v Z_{k}=-\gamma \cos \left(\eta_{k}\right), \\
\alpha v Z_{k}=\eta_{k} h^{-1}-\omega_{0}+\gamma \sin \left(\eta_{k}\right) .
\end{gathered}
$$

Отметим, что точки пересечения соответствующих кривых на плоскости $(\eta, Z)$ лежат на эллипсе, изображенном на рис. 1. Аналитически он задается уравнением

$$
(v Z)^{2}+\left(\alpha v Z-\eta h^{-1}+\omega_{0}\right)^{2}=\gamma^{2} .
$$

С другой стороны, исключая из (9), (10) переменную $Z$, приходим к трансцендентному уравнению

$$
\eta-\omega_{0} h=-\gamma h \sqrt{1+\alpha^{2}} \sin (\eta+\operatorname{arctg}(\alpha)),
$$

которое имеет по крайней мере одно решение. Легко видеть, что чем больше значение $\gamma h \sqrt{1+\alpha^{2}}$, тем больше решений имеет уравнение (12). Вычислив $\eta_{k}$, затем последовательно можем найти $\omega_{k}, Z_{k}, R_{k}$. В случае касания графиков правой и левой частей (12) происходит седло-узловая бифуркация рождения новой пары мода-антимода (см. $[24,28])$, которая возникает на плоскости $(\eta, Z)$ на пересечении эллипса с так называемой седло-узловой прямой

$$
v Z=\frac{1+\alpha \eta-\alpha \omega_{0} h}{h\left(1+\alpha^{2}\right)}
$$

Те моды на рис. 1, которые располагаются над седло-узловой прямой (13), называются антимодами или седловыми точками. Доказано, что они всегда неустойчивы. Собственно моды, лежащие ниже этой прямой, могут быть как устойчивыми, так и неустойчивыми. Величина обратной связи, при которой мода теряет устойчивость в результате бифуркации Андронова-Хопфа, приближенно оценивается следующим образом (см. [24]):

$$
\gamma_{k}^{H} \approx \frac{2 \lambda_{R}}{B_{k}\left[1-\cos \left(\omega_{R} h\right)\right]},
$$

где $\omega_{R}=\sqrt{2 v Q}$ задает угловую частоту, а $\lambda_{R}=(1+Q) / 2-$ скорость затухания колебаний (при этом их называют релаксационными) в отсутствие обратной связи,

$$
B_{k}=-\sqrt{1+\alpha^{2}} \cos \left(\eta_{k}-\operatorname{arctg} \alpha\right)
$$




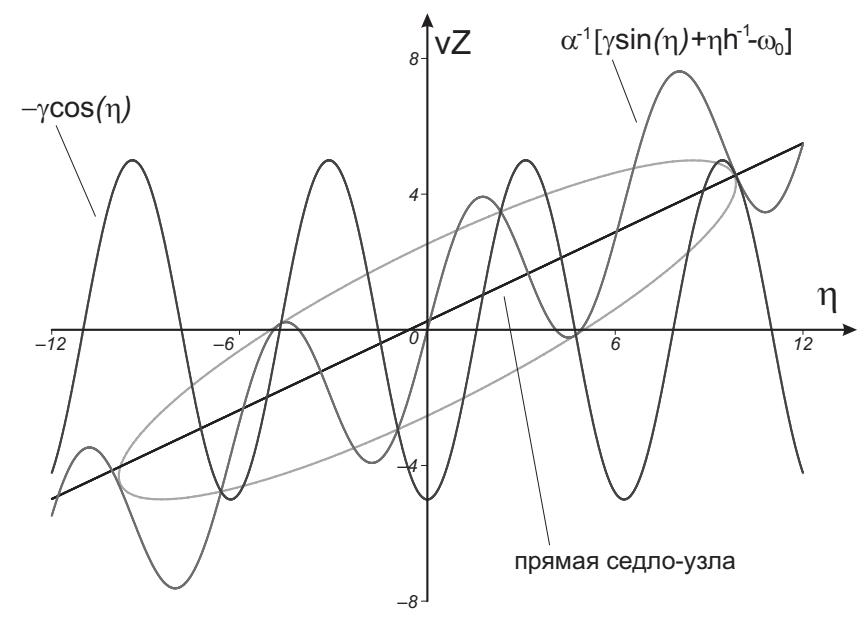

Рис. 1. Точки пересечения кривых (9) и (10) лежат на эллипсе (11) и соответствуют характеристикам $\left(\omega_{k}, Z_{k}\right)$ мод внешнего резонатора. Выбраны следующие значения параметров: $\alpha=1,7$; $\gamma=5 ; \omega_{0}=0 ; h=1$

В общем случае устойчивость мод внешнего резонатора определяется корнями характеристического уравнения:

$$
\begin{aligned}
{\left[\frac{1+Q}{1+Z_{k}}+\lambda\right]\left[\lambda^{2}+2 \gamma \cos \eta_{k}\left(1-e^{-\lambda h}\right) \lambda\right.} & \left.+\gamma^{2}\left(1-e^{-\lambda h}\right)^{2}\right]+ \\
& +2 v\left(Q-Z_{k}\right)\left[\lambda+\gamma\left(\cos \eta_{k}-\alpha \sin \eta_{k}\right)\left(1-e^{-\lambda h}\right)\right]=0
\end{aligned}
$$

Простейшие решения системы (3) при $n=1$ также определяются из соотношения (12) и обладают схожими свойствами, отмеченными в [8]. Замена

$$
E(t)=R_{E}(t) \cdot e^{i \theta_{E}(t)}, \quad f(t)=R_{f}(t) \cdot e^{i \theta_{f}(t)}
$$

приводит систему (4) к амплитудно-фазовому виду:

$$
\left\{\begin{array}{l}
\frac{d R_{E}}{d t}=v R_{E} Z+\gamma R_{f} \cos \left(\theta_{f}-\theta_{E}\right), \\
\frac{d \theta_{E}}{d t}=v \alpha Z+\gamma \frac{R_{f}}{R_{E}} \sin \left(\theta_{f}-\theta_{E}\right), \\
\frac{d R_{f}}{d t}=\Lambda\left[R_{E}(t-h) \cos \left(\theta_{E}(t-h)-\omega_{0} h-\theta_{f}\right)-R_{f}(t)\right], \\
\frac{d \theta_{f}}{d t}=\Delta+\Lambda \frac{R_{E}(t-h)}{R_{f}} \sin \left(\theta_{E}(t-h)-\omega_{0} h-\theta_{f}\right), \\
\frac{d Z}{d t}=Q-Z-(1+Z) R_{E}^{2} .
\end{array}\right.
$$

В данном случае простейшие решения (4) определяются из условий

$$
E(t)=E_{k} \cdot e^{i \omega_{k} t}, \quad f(t)=f_{k} \cdot e^{i \omega_{k} t+i \phi_{k}}, \quad Z(t)=Z_{k} .
$$

Такие периодические решения по аналогии с модами внешнего резонатора называются в [17] модами внешнего фильтра (external filtered modes, EFMs). В обозначениях системы (16) они имеют вид

$$
R_{E}(t)=E_{k}, \quad \theta_{E}(t)=\omega_{k} t, \quad R_{f}(t)=f_{k}, \quad \theta_{f}(t)=\omega_{k} t+\phi_{k}, \quad Z(t)=Z_{k},
$$


где $R_{k}, f_{k}, \omega_{k}, \phi_{k}, Z_{k}$ - константы. Подставляя найденные выражения в (16), получим уравнения

$$
\left\{\begin{array}{l}
0=v E_{k} Z_{k}+\gamma \cos \left(\phi_{k}\right) f_{k} \\
\omega_{k}=v \alpha Z_{k}+\gamma \sin \left(\omega_{k}\right) \frac{f_{k}}{E_{k}} \\
0=\Lambda\left[E_{k} \cos \left(-\omega_{0} h-\omega_{k} h-\phi_{k}\right)-f_{k}\right] \\
\omega_{k}=\Delta+\Lambda \sin \left(-\omega_{0} h-\omega_{k} h-\phi_{k}\right) \frac{E_{k}}{f_{k}} \\
0=Q-Z_{k}-\left(1+Z_{k}\right) E_{k}^{2}
\end{array}\right.
$$

из которых, полагая $E_{k} \neq 0$, можно получить уравнение относительно величины $\omega_{k}$ :

$$
\omega_{k}=-\gamma \sqrt{1+\alpha^{2}} \frac{\Lambda}{\sqrt{\Lambda^{2}+\left(\Delta-\omega_{k}\right)^{2}}} \sin \left(\omega_{0} h+\omega_{k} h+\operatorname{arctg} \alpha+\operatorname{arctg}\left(\frac{\Delta-\omega_{k}}{\Lambda}\right)\right) .
$$

Трансцендентное уравнение (18) является основным при определении мод внешнего фильтра. Величины $\phi_{k}$ и $\omega_{k}$ связаны соотношением

$$
\phi_{k}=-\omega_{0} h-\omega_{k} h-\operatorname{arctg}\left(\frac{\Delta-\omega_{k}}{\Lambda}\right) .
$$

По $\phi_{k}$ и $\omega_{k}$ определяются остальные характеристики мод:

$$
v Z_{k}=-\gamma \cos \left(\phi_{k}\right) \frac{\Lambda}{\sqrt{\Lambda^{2}+\left(\Delta-\omega_{k}\right)^{2}}}, \quad E_{k}^{2}=\frac{Q-Z_{k}}{1+Z_{k}}, \quad f_{k}^{2}=\frac{\Lambda^{2} E_{k}^{2}}{\Lambda^{2}+\left(\Delta-\omega_{k}\right)^{2}} .
$$

Если в правой части формулы (18) множитель, задаваемый функцией sin принимает значения \pm 1 , то (18) переходит в уравнение $P\left(\omega_{k}\right)=0$, где

$$
P\left(\omega_{k}\right)=\left[\Lambda^{2}+\left(\Delta-\omega_{k}\right)^{2}\right] \omega_{k}^{2}-\Lambda^{2} \gamma^{2}\left(1+\alpha^{2}\right) .
$$

Его решения задают границу области существования решений (18).

В пределе при $\Lambda \rightarrow \infty$, когда фильтр пропускает весь спектр излучения, уравнение (18) переходит в уравнение (12), а моды внешнего фильтра совпадают с модами внешнего резонатора.

4. Методика исследования на примере моделей одномодового лазера. Проиллюстрируем сначала методику исследования на более простых моделях одномодового лазера. Частично результаты данного раздела представлены в [3]. Результаты для моделей многомодового лазера в разделах 6-8 являются новыми.

Рассмотрим уравнение (1) при условии, что $Q=\varepsilon^{-1}$, где $\varepsilon$-малый положительный параметр. Нормируя переменные следующим образом:

$$
t=\varepsilon s, \quad E=\frac{1}{\sqrt{\varepsilon}} \cdot e^{-i \omega s} \tilde{E},
$$

приведем исходную систему к виду

$$
\left\{\begin{array}{l}
\frac{d \tilde{E}}{d s}=i \omega \tilde{E}+\varepsilon v(1+i \alpha) \tilde{E} Z+\varepsilon \gamma e^{-i \omega_{0} h+i \omega h \varepsilon^{-1}} \tilde{E}\left(s-h \varepsilon^{-1}\right), \\
\frac{d Z}{d s}=1-(1+Z)|\tilde{E}|^{2}-\varepsilon Z
\end{array}\right.
$$

который является частным случаем следующего векторного уравнения:

$$
x^{\prime}=F(x)+\varepsilon \cdot \Phi\left(x, x\left(s-h \varepsilon^{-1}\right)\right) .
$$

Общая методика исследования его динамики была предложена в [5]. В соответствии с представленным там алгоритмом, в малой окрестности некоторого периодического решения системы 
«нулевого приближения» $x^{\prime}=F(x)$ строится нормализующая замена, позволяющая получить асимптотические оценки системы (21). Эта замена имеет следующий вид:

$$
\begin{gathered}
V(s, \varepsilon)=V_{0}(\tau)+\varepsilon V_{1}(t, \tau)+\varepsilon^{2} V_{2}(t, \tau)+\ldots, \\
\frac{d \tau}{d s}=1+\varepsilon \varphi(t)+\varepsilon^{2} \psi(t)+\ldots
\end{gathered}
$$

Здесь $V_{j}(t, \tau)$ являются $T$-периодичными по $\tau$, причем $V_{0}(s)$ есть периодическое решение системы ОДУ $x^{\prime}=F(x), \varphi(t)$ - скалярная почти периодическая функция.

Отметим, что из (23) следует, что

$$
\tau\left(s-h \varepsilon^{-1}\right) \approx \tau(s)-y, \quad \text { где } \quad y=h \varepsilon^{-1}+\int_{-h}^{0} \varphi(t+r) d r .
$$

Стандартная линеаризация $x^{\prime}=F(x)$ на $V_{0}(s)$ приводит к системе

$$
u^{\prime}=A(s) u,
$$

где $A(s)=F^{\prime}\left(V_{0}(s)\right)$ есть $T$-периодическая матрица. Обозначим через $H_{j}(s)$ линейно независимые периодические решения сопряженной к $(24)$ системы $w^{\prime}=-A^{*}(s) w$.

Подставляя ряды $(22),(23)$ в (21) и приравнивая коэффициенты при одинаковых степенях $\varepsilon$, получим цепочку уравнений вида

$$
\frac{d V_{k}}{d \tau}=A(\tau) V_{k}+f_{k}(t, \tau, \tau-y), \quad k \in \mathbb{N},
$$

где через $f_{k}(t, \tau, \tau-y)$ обозначена неоднородность дифференциального уравнения. На первом шаге из условий периодичности функции $V_{1}$ по $\tau$ получим систему уравнений

$$
\left\langle f_{1}(t, \tau, \tau-y), H_{j}(\tau)\right\rangle=0,
$$

где угловыми скобками $\langle\cdot, \cdot\rangle$ обозначено скалярное произведение

$$
\langle X, Y\rangle=\frac{1}{T} \int_{0}^{T}(X(\tau), Y(\tau)) d \tau .
$$

Полученные уравнения (26) играют роль нормальной формы в задаче о локальной динамике уравнения $(21)$ в некоторой окрестности решения $V_{0}(s)$ системы «нулевого приближения» $x^{\prime}=$ $F(x)$. Отыскав решение $V_{1}$ системы $(25)$ в указанном классе функций, можно последовательно найти любое число элементов рядов $(22),(23)$.

В случае уравнений Ланга-Кобаяси при $Q \gg 1$ система ОДУ вида $x^{\prime}=F(x)$ является консервативной и имеет семейство периодических решений, которое в форме вектора с вещественными компонентами может быть записано следующим образом:

$$
V_{0}(s)=\left(c \cos (\omega s), c \sin (\omega s), c^{-2}-1\right)^{T} .
$$

Матрица системы (24) имеет вид

$$
A(s)=F^{\prime}\left(V_{0}(s)\right)=\left(\begin{array}{ccc}
0 & -\omega & 0 \\
\omega & 0 & 0 \\
-2 c^{-1} \cos (\omega s) & -2 c^{-1} \sin (\omega s) & -c^{2}
\end{array}\right) .
$$

Для системы (24) легко находятся все линейно независимые решения, два из которых можно выбрать периодическими с периодом $T=2 \pi / \omega$ :

$$
K_{0}(s)=\left(\begin{array}{c}
\cos (\omega s) \\
\sin (\omega s) \\
-2 c^{-3}
\end{array}\right), \quad K_{1}(s)=\left(\begin{array}{c}
-\sin (\omega s) \\
\cos (\omega s) \\
0
\end{array}\right), \quad K_{2}(s)=\left(\begin{array}{c}
0 \\
0 \\
e^{-c^{2} s}
\end{array}\right) .
$$

Очевидно, мультипликаторы системы (24) имеют вид

$$
\mu_{1}=\mu_{2}=1, \quad \mu_{3}=e^{-\frac{2 \pi}{\omega} c^{2}}<1 .
$$


Линейно независимые периодические решения сопряженной к (24) системы выберем таким образом, чтобы $\left\langle K_{i}, H_{j}\right\rangle=\delta_{i j}, i, j=0,1$, где $\delta_{i j}$ - символ Кронекера. Таким условиям удовлетворяют вектор-функции вида

$$
H_{0}(s)=\left(\begin{array}{c}
\cos (\omega s) \\
\sin (\omega s) \\
0
\end{array}\right), \quad H_{1}(s)=\left(\begin{array}{c}
-\sin (\omega s) \\
\cos (\omega s) \\
0
\end{array}\right) .
$$

Рассмотрим свободную постоянную $c$ как функцию «медленного» времени: $t=\varepsilon s$. В соответствии с изложенной методикой, собирая коэффициенты при первой степени $\varepsilon$, придем к системе

$$
c^{\prime}(t) \frac{d V_{0}}{d c}+\varphi(t) \frac{d V_{0}}{d \tau}+\frac{d V_{1}}{d \tau}=A(\tau) V_{1}+\Phi\left(V_{0}(\tau), V_{0}(\tau-y)\right) .
$$

Условие существования $2 \pi / \omega$-периодического (по $\tau$ ) решения уравнения $(27)$ состоит в ортогональности его неоднородной части функциям $H_{0}(\tau), H_{1}(\tau)$. Выполнив ряд преобразований и переобозначив $\omega \varphi \rightarrow \varphi$, получим следующую систему интегро-дифференциальных уравнений, которую мы будем называть квазинормальной формой модели Ланга-Кобаяси в случае $Q \gg 1$ :

$$
\left\{\begin{array}{l}
c^{\prime}(t)=v\left(\frac{1}{c(t)}-c(t)\right)+\gamma \cos \left(\omega_{0} h+\int_{-h}^{0} \varphi(t+r) d r\right) c(t-h), \\
\varphi(t)=v \alpha\left(\frac{1}{c^{2}(t)}-1\right)-\gamma \sin \left(\omega_{0} h+\int_{-h}^{0} \varphi(t+r) d r\right) \frac{c(t-h)}{c(t)} .
\end{array}\right.
$$

Заметим, что система (28) заменами

$$
\varphi(t)=\theta^{\prime}(t), \quad c(t) e^{i \theta(t)}=E(t)
$$

может быть преобразована к одному комплексному уравнению следующего вида:

$$
\frac{d E}{d t}=v(1+i \alpha)\left(|E|^{-2}-1\right) E+\gamma e^{-i \omega_{0} h} E(t-h)
$$

которое, очевидно, связано с (1).

Более простой и быстрый способ получения этого результата дает постановка исходной задачи в терминах системы уравнений (7). Наряду с (20) рассмотрим систему

$$
\left\{\begin{array}{l}
\frac{d \tilde{R}}{d s}=\varepsilon v Z \tilde{R}+\varepsilon \gamma \cos \left(\omega_{0} h-\omega h \varepsilon^{-1}+\tilde{\theta}-\tilde{\theta}\left(s-h \varepsilon^{-1}\right)\right) \tilde{R}\left(s-h \varepsilon^{-1}\right), \\
\frac{d \tilde{\theta}}{d s}=\omega+\varepsilon v \alpha Z-\varepsilon \gamma \sin \left(\omega_{0} h-\omega h \varepsilon^{-1}+\tilde{\theta}-\tilde{\theta}\left(s-h \varepsilon^{-1}\right)\right) \frac{\tilde{R}\left(s-h \varepsilon^{-1}\right)}{\tilde{R}} \\
\frac{d Z}{d s}=1-(1+Z) \tilde{R}^{2}-\varepsilon Z
\end{array}\right.
$$

с периодической по $\tilde{\theta}$ и $\tilde{\theta}\left(s-h \varepsilon^{-1}\right)$ правой частью, решение которой в «нулевом приближении» имеет вид

$$
V_{0}(s)=\left(c, \omega s, c^{-2}-1\right)^{T} .
$$

Оно периодично в силу периодичности правой части (30) по переменной $\tilde{\theta}$.

Стандартная линеаризация $x^{\prime}=F(x)$ на $V_{0}(s)$ в соответствии с изложенной методикой приводит к системе $(24)$ с постоянной матрицей

$$
A=\frac{D F}{D x}\left(V_{0}(s)\right)=\left(\begin{array}{ccc}
0 & 0 & 0 \\
0 & 0 & 0 \\
-2 c^{-1} & 0 & -c^{2}
\end{array}\right) .
$$

Очевидно, что среди трех ее вещественных собственных значений есть два нулевых и одно вещественное отрицательное $-c^{2}$. Для системы (24) в данном случае также находятся все линейно независимые решения, два из которых можно выбрать периодическими: $K_{0}(s)=\left(1,0,-2 c^{-3}\right)^{T}$, 
$K_{1}(s)=(0,1,0)^{T}, K_{2}(s)=\left(0,0, e^{-c^{2} s}\right)^{T}$. Полученные линейно независимые решения позволяют утверждать, что мультипликаторы системы (24) имеют вид $\mu_{1}=\mu_{2}=1,\left|\mu_{3}\right|<1$.

Аналогично находятся линейно независимые периодические решения сопряженной к (24) системы. Выберем их следующим образом: $H_{0}(s)=(1,0,0)^{T}, H_{1}(s)=(0,1,0)^{T}$. Далее точно так же требуется ортогональность неоднородной части уравнения $(27)$ функциям $H_{0}(\tau), H_{1}(\tau)$. При этом процесс вычислений значительно упрощается, поэтому в других случаях будем пользоваться такой параметризацией из соображений удобства.

Рассмотрим систему (3) в случае $n=1$, т.е. одномодовый вариант модели, где $E_{1}$ и $Z_{1}$ будут обозначаться $E$ и $Z$ :

$$
\left\{\begin{array}{l}
\frac{d E}{d t}=v(1+i \alpha)\left[\gamma_{1}\left(Z_{0}-Z\right)-1\right] E+\gamma e^{-i \Omega h} E(t-h) \\
\frac{d Z}{d t}=-\left(1+d+\gamma_{1}|E|^{2}\right) Z+\gamma_{1}|E|^{2} Z_{0} / 2 \\
\frac{d Z_{0}}{d t}=Q-Z_{0}+|E|^{2}\left(Z-Z_{0}\right) .
\end{array}\right.
$$

Выполняя аналогичные преобразования, получаем следующий частный случай системы (21):

$$
\left\{\begin{array}{l}
\frac{d \tilde{E}}{d s}=i \omega \tilde{E}+\varepsilon v(1+i \alpha)\left[\gamma_{1}\left(Z_{0}-Z\right)-1\right] \tilde{E}+\varepsilon \gamma e^{-i \Omega h+i \omega h \varepsilon^{-1}} \tilde{E}\left(s-h \varepsilon^{-1}\right), \\
\frac{d Z}{d s}=\gamma_{1}|\tilde{E}|^{2} Z_{0} / 2-\gamma_{1}|\tilde{E}|^{2} Z-\varepsilon(1+d) Z \\
\frac{d Z_{0}}{d s}=1+|\tilde{E}|^{2}\left(Z-Z_{0}\right)-\varepsilon Z_{0} .
\end{array}\right.
$$

Решение системы нулевого приближения в данном случае может быть записано как

$$
V_{0}(s)=\left(c \cos (\omega s), c \sin (\omega s), c^{-2}, 2 c^{-2}\right)^{T},
$$

или при переходе от комплексной амплитуды $E$ к вещественным переменным $R$ и $\theta$ как

$$
V_{0}(s)=\left(c, \omega s, c^{-2}, 2 c^{-2}\right)^{T} .
$$

Стандартная линеаризация $x^{\prime}=F(x)$ на $V_{0}(s)$ в соответствии с изложенной методикой приводит к системе $(24)$ с постоянной матрицей

$$
A=\frac{D F}{D x}\left(V_{0}(s)\right)=\left(\begin{array}{cccc}
0 & 0 & 0 & 0 \\
0 & 0 & 0 & 0 \\
0 & 0 & -\gamma_{1} c^{2} & \gamma_{1} c^{2} / 2 \\
-2 c^{-1} & 0 & c^{2} & -c^{2}
\end{array}\right)
$$

Среди собственных значений этой матрицы очевидно есть два нулевых, которым соответствуют следующие линейно независимые решения: $K_{0}(s)=\left(1,0,-2 c^{-3},-4 c^{-3}\right)^{T}, K_{1}(s)=(0,1,0,0)^{T}$. Еще два собственных значения матрицы $A$ являются собственными значениями подматрицы

$$
B_{1}=\left(\begin{array}{cc}
-\gamma_{1} c^{2} & \gamma_{1} c^{2} / 2 \\
c^{2} & -c^{2}
\end{array}\right) .
$$

Непосредственные вычисления показывают, что они вещественные отрицательные.

Линейно независимые периодические решения сопряженной системы удобно выбрать следующим образом: $H_{0}(s)=(1,0,0,0)^{T}, H_{1}(s)=(0,1,0,0)^{T}$. Из условий $(26)$, переобозначая $\omega \varphi \rightarrow \varphi$, получим следующую систему интегро-дифференциальных уравнений, которую мы будем называть квазинормальной формой модели (31) в случае $Q \gg 1$ : 


$$
\left\{\begin{array}{l}
c^{\prime}(t)=v\left(\frac{\gamma_{1}}{c(t)}-c(t)\right)+\gamma \cos \left(\Omega h+\int_{-h}^{0} \varphi(t+r) d r\right) c(t-h), \\
\varphi(t)=v \alpha\left(\frac{\gamma_{1}}{c^{2}(t)}-1\right)-\gamma \sin \left(\Omega h+\int_{-h}^{0} \varphi(t+r) d r\right) \frac{c(t-h)}{c(t)} .
\end{array}\right.
$$

Заметим, что система (33) заменами $\varphi(t)=\theta^{\prime}(t), c(t) e^{i \theta(t)}=E(t)$ может быть преобразована к одному комплексному уравнению

$$
\frac{d E}{d t}=v(1+i \alpha)\left(\gamma_{1}|E|^{-2}-1\right) E+\gamma e^{-i \Omega h} E(t-h),
$$

которое нормировочной заменой $\gamma_{1}^{-1 / 2} E \rightarrow E$ и переобозначением $\Omega \rightarrow \omega_{0}$ приводится к виду (29).

Таким образом, можно говорить о том, что динамика обеих моделей (2) и (3) при $n=1$ в случае $Q \gg 1$ математически описывается одним и тем же уравнением, и в этом смысле они не отличаются друг от друга. Это соответствует результатам, сформулированным в работе [8], где отличия между моделями фиксируются в режимах низкочастотных флуктуаций, возникающих при малых величинах тока накачки $(0<Q \ll 1)$, а также малых значениях коэффициента диффузии $d$, который в рассматриваемом случае исключается.

5. Динамические свойства упрощенной модели. Стационарные решения системы (28) определяются из трансцендентного уравнения относительно $\varphi$

$$
\varphi=-\gamma\left[\alpha \cos \left(\omega_{0} h+\varphi h\right)+\sin \left(\omega_{0} h+\varphi h\right)\right]
$$

Обозначая $\eta=\omega_{0} h+\varphi h$, придем к хорошо известному соотношению (12) для определения числа мод внешнего резонатора.

Отметим, что по $\eta_{k}$ можно однозначно определить $\varphi$ и $c$. Устойчивость каждого из состояний равновесия системы (28) определяется расположением в комплексной плоскости корней характеристического квазиполинома

$$
\lambda^{2}+2 \lambda \gamma \cos \eta_{k}\left(1-e^{-\lambda h}\right)+\gamma^{2}\left(1-e^{-\lambda h}\right)^{2}+2\left(v-\gamma \cos \eta_{k}\right)\left[\lambda+\gamma\left(\cos \eta_{k}-\alpha \sin \eta_{k}\right)\left(1-e^{-\lambda h}\right)\right] .
$$

В случае $\cos \eta_{k}=1$ для моды с максимальным усилением [24] уравнение (35) сводится к следующей простой совокупности:

$$
\left[\begin{array}{l}
\lambda+\gamma\left(1-e^{-\lambda h}\right)=0 \\
\lambda+\gamma\left(1-e^{-\lambda h}\right)+2(v-\gamma)=0 .
\end{array}\right.
$$

При $0<\gamma<v$ у каждого из уравнений (36) отсутствуют корни с положительной вещественной частью. Следовательно, мода с максимальным усилением в случае асимптотически большой накачки является устойчивой. Этот результат хорошо согласуется с выводами, сделанными в [24].

Мода с минимальной шириной линии достигается при $\eta_{k}=-\operatorname{arctg}(\alpha)$. Достаточным условием ее устойчивости является следующая теорема.

Теорема 1. Пусть

$$
\eta_{k}=-\operatorname{arctg}(\alpha), \quad \gamma \leqslant \gamma_{1}=\frac{v}{\sqrt{1+\alpha^{2}}} .
$$

Тогда все корни уравнения (35) имеют неположительные действительные части.

Критерием устойчивости моды с минимальной шириной линии в случае большого запаздывания $h=\mu^{-1}$, где $\mu$ - малый параметр, является следующая теорема.

Теорема 2. Пусть

$$
\eta_{k}=-\operatorname{arctg}(\alpha), \quad \gamma<\gamma_{2}=\frac{2 v \sqrt{1+\alpha^{2}}}{2+\alpha^{2}}
$$




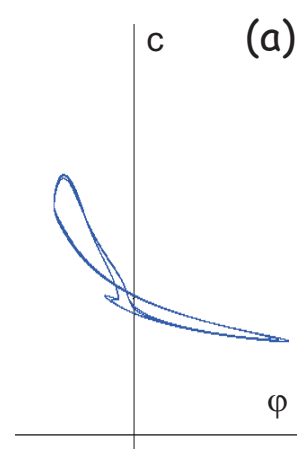

(a)

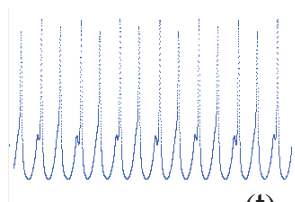

$\varphi(\mathrm{t})$

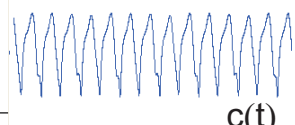

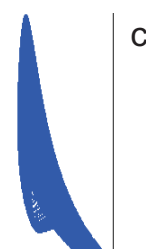

c

(b)

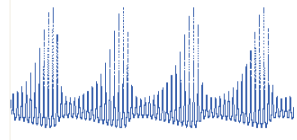

$\varphi(\mathrm{t})$

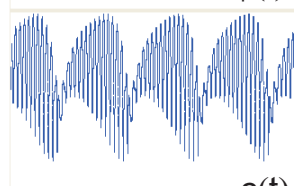

Рис. 2. Иллюстрация явления мультистабильности. При значениях параметров $v=100, \alpha=$ $5, \omega_{0} h=1, \gamma=97,9, h=0,04$ найдено три различных устойчивых режима: мода, близкая к состоянию с максимальным усилением, цикл удвоенного периода (а) и режим, известный как RPP (regular pulse package) (b) (cm. [20,33]).
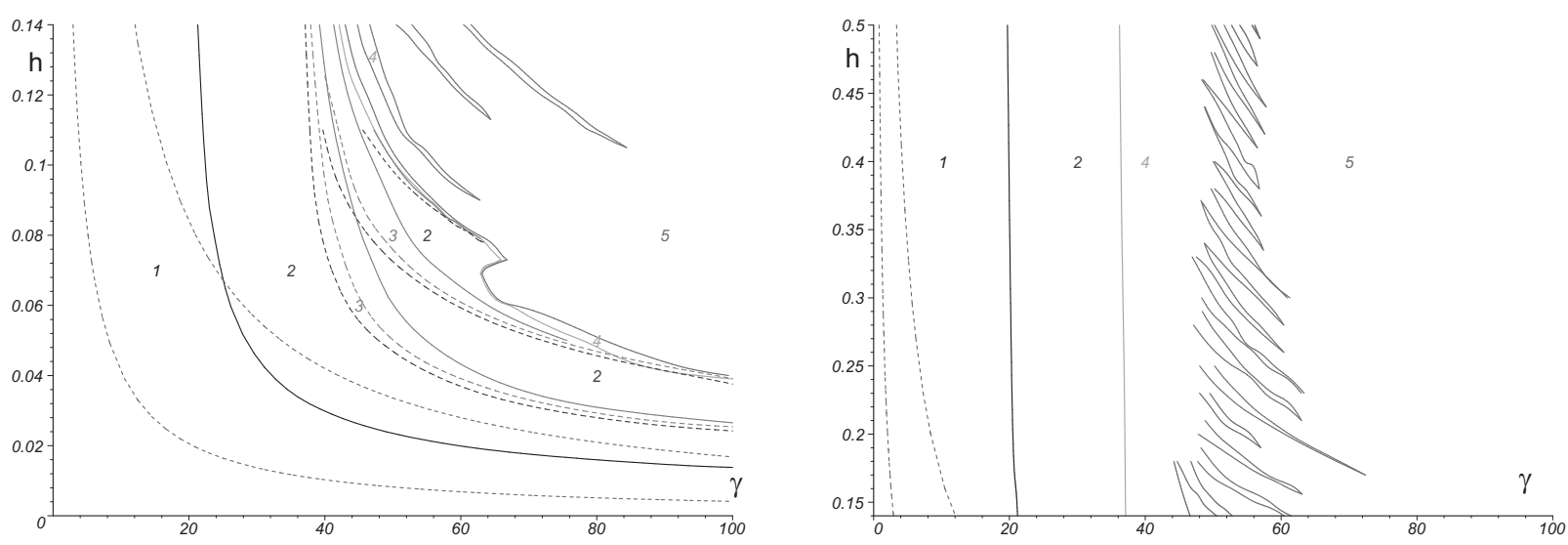

Рис. 3. Бифуркационная диаграмма системы $(28)$ на плоскости $(\gamma, h)$, разделенная на две части с разным масштабом по параметру $h$. Отметим существование областей мультистабильности.

Тогда все корни уравнения (35) имеют неположительные действительные части. В случае $\gamma>\gamma_{2}$ существует корень с положительной действительной частью.

Непосредственной подстановкой несложно проверить, что корней с асимптотикой $i \omega_{*} \mu^{-p}$, где $p>0$, у уравнения (35) в случае $\eta_{k}=-\operatorname{arctg}(\alpha)$ нет. Раскладывая $\lambda$ в ряд по степеням малого параметра $\mu$, так что $\lambda=\lambda_{0}+\mu \lambda_{1}+\mu^{2} \lambda_{2}+\ldots$, получаем $\operatorname{Re} \lambda_{0}=\operatorname{Re} \lambda_{1}=0$, а знак $\operatorname{Re} \lambda_{2}$ определяет знак выражения $\gamma\left(2+\alpha^{2}\right)-2 v \sqrt{1+\alpha^{2}}$.

Основные результаты относительно динамических свойств системы (28) получены численным моделированием. При этом исследовалась не сама система (28), а система дифференциальных уравнений с запаздыванием, получаемая заменой $\varphi(t)=\theta^{\prime}(t)$. Тем не менее, в качестве основных характеристик модифицированной таким образом квазинормальной формы выступали функции $c(t), \theta^{\prime}(t)$.

Численное интегрирование выполнялось с помощью схемы Рунге-Кутты четвертого порядка, адаптированной для систем с запаздыванием. В качестве начальных условий выбирались функции из класса тригонометрических полиномов, заданных на отрезке $[-h, 0]$. Были обнаружены явления мультистабильности (рис. 2), кризиса аттракторов, последовательного удвоения периода циклов, определены области с хаотической и сложной нехаотической динамикой.

Важные особенности динамики квазинормальной формы (28) отражает бифуркационная диаграмма, представленная на рис. 3. Выбраны следующие значения параметров $v=100, \alpha=5$, 
$\omega_{0} h=1$. Цифрами обозначены области: 1 - состояние равновесия; 2 - цикл, рождающийся в результате бифуркации Андронова-Хопфа; 3 - сценарий Фейгенбаума - каскад бифуркаций удвоения периода (пунктиром отмечены первые две бифуркации удвоения); 4-нефейгенбаумовские переходные режимы (в некоторых случаях интерпретировались как квазипериодические или двухчастотные на основе анализа временного ряда стандартной функцией FFT пакета Maple); 5 хаос. Штрихпунктиром обозначены первые две бифуркации седло-узла, в которых происходит рождение пары мода-антимода. Правее первой из них существуют области мультистабильности. Изменение бассейнов притяжения лежит в основе перехода (при возрастании параметров $h, \gamma$ ) от все усложняющихся режимов из области 3 к области 2. Область, отмеченная цифрой 4 на верхней части диаграммы, имеет сложную структуру и содержит многочисленные участки типа 2 и 5 малых размеров. Отметим, что существование устойчивого состояния равновесия, близкого к моде с максимальным усилением, подтверждается численно. При $\gamma>v \sqrt{1+\alpha^{2}}$ система (1), вообще говоря, теряет свойство диссипативности. Это объясняется тем, что столь большие значения $\gamma$ физически недостижимы, поскольку величину $v^{-1} \gamma$ следует понимать как ту долю отраженного излучения, которая возвращается в резонатор.

Граница области динамического хаоса определялась на основе вычисления корреляционной размерности (см. $[15,16])$. Расчет старшего ляпуновского показателя (см. [36]) в целом подтверждает полученные оценки. Таким образом, наблюдаемая динамика системы (28) хорошо согласуется с известными о когерентном коллапсе фактами (см. [19,30,34,37]).

Подчеркнем связь между установившимися режимами в квазинормальной форме и в исходной модели: устойчивым состояниям равновесия и циклам системы (28) соответствуют устойчивые предельные циклы и торы в (1).

6. Результаты для моделей многомодового лазера. Для модели (2) в результате аналогичных действий

$$
t=\varepsilon s, \quad E_{m}=\frac{1}{\sqrt{\varepsilon}} \cdot e^{-i \omega s} \tilde{E}_{m}
$$

приходим к следующему частному случаю (21):

$$
\left\{\begin{array}{l}
\frac{d \tilde{E}_{m}}{d s}=i \omega \tilde{E}_{m}+\varepsilon v(1+i \alpha) \tilde{E}_{m} Z+\varepsilon \gamma e^{-i \Omega_{m} h+i \omega h \varepsilon^{-1}} \tilde{E}_{m}\left(s-h \varepsilon^{-1}\right), \\
\frac{d Z}{d s}=1-(1+Z) \sum_{j=1}^{n}\left|\tilde{E}_{j}\right|^{2}-\varepsilon Z
\end{array}\right.
$$

При $n=2$ семейство периодических решений системы нулевого приближения можно записать в форме вектора с вещественными компонентами:

где

$$
V_{0}(s)=\left(c_{1} \cos (\omega s), c_{1} \sin (\omega s), Z_{1}, c_{2} \cos (\omega s), c_{2} \sin (\omega s), Z_{2}\right)^{T},
$$

$$
Z_{1}=Z_{2}=\frac{1}{\rho_{2}}-1, \quad \rho_{2}=c_{1}^{2}+c_{2}^{2},
$$

или в случае замены в $(37)$ величины $\tilde{E}_{m}=\tilde{R}_{m} \exp \left(i \tilde{\theta}_{m}\right)$ в виде

$$
V_{0}(s)=\left(c_{1}, \omega s, Z_{1}, c_{2}, \omega s, Z_{2}\right)^{T} \text {. }
$$

Стандартная линеаризация $x^{\prime}=F(x)$ на $V_{0}(s)$ в соответствии с изложенной методикой приводит к системе $(24)$ с постоянной матрицей

$$
A=\frac{D F}{D x}\left(V_{0}(s)\right)=\left(\begin{array}{cccccc}
0 & 0 & 0 & 0 & 0 & 0 \\
0 & 0 & 0 & 0 & 0 & 0 \\
-2 c_{1} / \rho_{2} & 0 & -\rho_{2} & -2 c_{2} / \rho_{2} & 0 & 0 \\
0 & 0 & 0 & 0 & 0 & 0 \\
0 & 0 & 0 & 0 & 0 & 0 \\
-2 c_{1} / \rho_{2} & 0 & 0 & -2 c_{2} / \rho_{2} & 0 & -\rho_{2}
\end{array}\right)
$$


где выполнены замены $\rho_{2}=c_{1}^{2}+c_{2}^{2}$ и $1+Z_{i}=1 / \rho_{2}, i=1,2$. Среди собственных значений этой матрицы очевидно есть четыре нулевых (и два вещественных отрицательных), которым соответствуют линейно независимые решения $K_{i}(s), i=0, \ldots, 5$, записанные по столбцам матричной экспоненты:

$$
\exp (A s)=\left(\begin{array}{cccccc}
1 & 0 & 0 & 0 & 0 & 0 \\
0 & 1 & 0 & 0 & 0 & 0 \\
-2 c_{1}\left(1-e^{-\rho_{2} s}\right) / \rho_{2}^{2} & 0 & e^{-\rho_{2} s} & -2 c_{2}\left(1-e^{-\rho_{2} s}\right) / \rho_{2}^{2} & 0 & 0 \\
0 & 0 & 0 & 1 & 0 & 0 \\
0 & 0 & 0 & 0 & 1 & 0 \\
-2 c_{1}\left(1-e^{-\rho_{2} s}\right) / \rho_{2}^{2} & 0 & 0 & -2 c_{2}\left(1-e^{-\rho_{2} s}\right) / \rho_{2}^{2} & 0 & e^{-\rho_{2} s}
\end{array}\right) .
$$

Выберем следующие линейно независимые периодические решения сопряженной системы: $H_{0}(s)=(1,0,0,0,0,0)^{T}, H_{1}(s)=(0,1,0,0,0,0)^{T}, H_{3}(s)=(0,0,0,1,0,0)^{T}, H_{4}(s)=(0,0,0,0,1,0)^{T}$. В данном случае замена $(22),(23)$ несколько модифицируется:

$$
\begin{gathered}
V(s, \varepsilon)=V_{0}\left(\tau_{1}, \tau_{2}\right)+\varepsilon V_{1}\left(t, \tau_{1}, \tau_{2}\right)+\varepsilon^{2} V_{2}\left(t, \tau_{1}, \tau_{2}\right)+\ldots, \\
\frac{d \tau_{1}}{d s}=1+\varepsilon \varphi_{1}(t)+\varepsilon^{2} \psi_{1}(t)+\ldots, \quad \frac{d \tau_{2}}{d s}=1+\varepsilon \varphi_{2}(t)+\varepsilon^{2} \psi_{2}(t)+\ldots
\end{gathered}
$$

Здесь $V_{0}\left(\tau_{1}, \tau_{2}\right)=\left(c_{1} \exp \left(i \omega \tau_{1}\right), Z_{1}, c_{2} \exp \left(i \omega \tau_{2}\right), Z_{2}\right)^{T}$. Из условий $(26)$, переобозначая $\omega \varphi_{j} \rightarrow \varphi_{j}$, получим следующую систему интегро-дифференциальных уравнений, которую мы будем называть квазинормальной формой модели (2) в случае $Q \gg 1$ :

$$
\left\{\begin{array}{l}
c_{1}^{\prime}(t)=v\left(\frac{1}{c_{1}^{2}(t)+c_{2}^{2}(t)}-1\right) c_{1}(t)+\gamma \cos \left(\Omega_{1} h+\int_{-h}^{0} \varphi_{1}(t+r) d r\right) c_{1}(t-h) \\
\varphi_{1}(t)=v \alpha\left(\frac{1}{c_{1}^{2}(t)+c_{2}^{2}(t)}-1\right)-\gamma \sin \left(\Omega_{1} h+\int_{-h}^{0} \varphi_{1}(t+r) d r\right) \frac{c_{1}(t-h)}{c_{1}(t)} \\
c_{2}^{\prime}(t)=v\left(\frac{1}{c_{1}^{2}(t)+c_{2}^{2}(t)}-1\right) c_{2}(t)+\gamma \cos \left(\Omega_{2} h+\int_{-h}^{0} \varphi_{2}(t+r) d r\right) c_{2}(t-h) \\
\varphi_{2}(t)=v \alpha\left(\frac{1}{c_{1}^{2}(t)+c_{2}^{2}(t)}-1\right)-\gamma \sin \left(\Omega_{2} h+\int_{-h}^{0} \varphi_{2}(t+r) d r\right) \frac{c_{2}(t-h)}{c_{2}(t)}
\end{array}\right.
$$

Заметим, что система (38) заменами

$$
\varphi_{j}(t)=\theta_{j}^{\prime}(t), \quad c_{j}(t) e^{i \theta_{j}(t)}=E_{j}(t), \quad j=1,2,
$$

может быть преобразована к системе из двух комплексных уравнений

$$
\left\{\begin{array}{l}
\frac{d E_{1}}{d t}=v(1+i \alpha)\left[\frac{1}{\left|E_{1}\right|^{2}+\left|E_{2}\right|^{2}}-1\right] E_{1}+\gamma e^{-i \Omega_{1} h} E_{1}(t-h), \\
\frac{d E_{2}}{d t}=v(1+i \alpha)\left[\frac{1}{\left|E_{1}\right|^{2}+\left|E_{2}\right|^{2}}-1\right] E_{2}+\gamma e^{-i \Omega_{2} h} E_{2}(t-h) .
\end{array}\right.
$$

В общем случае при произвольном натуральном $n$ вектор $V_{0}(s)$ имеет $3 n$ вещественных компонент, которые удобно представить как $n$ блоков из троек $c_{m}, \omega s, Z_{m}$, где $Z_{m}=\left(1 / \rho_{n}\right)-1, \rho_{n}=c_{1}^{2}+\ldots+c_{n}^{2}$. Матрица $A$ тогда состоит из $n^{2}$ блоков размера $3 \times 3$, где главная диагональ занята подматрицами с двумя ненулевыми элементами, а остальные блоки имеют лишь один ненулевой элемент:

$$
\left(\begin{array}{ccc}
0 & 0 & 0 \\
0 & 0 & 0 \\
-2 c_{m} / \rho_{n} & 0 & -\rho_{n}
\end{array}\right), \quad\left(\begin{array}{ccc}
0 & 0 & 0 \\
0 & 0 & 0 \\
-2 c_{m} / \rho_{n} & 0 & 0
\end{array}\right), \quad \text { где } \quad \rho_{n}=\sum_{j=1}^{n} c_{j}^{2},
$$


а выписанные блоки образуют $m$-й «столбец» матрицы $A$. Среди собственных значений $A$ есть $2 n$ нулевых и $n$ вещественных отрицательных, равных $-\rho_{n}$. Нулевым собственным значениям матрицы $A$ соответствуют единичные мультипликаторы системы (24), число которых определяет число условий (26). Окончательный результат по аналогии со случаем $n=2$ можно сформулировать в форме обобщения системы (39) вида

$$
\frac{d E_{m}}{d t}=v(1+i \alpha)\left[\frac{1}{\sum_{j=1}^{n}\left|E_{j}\right|^{2}}-1\right] E_{m}+\gamma_{m} e^{-i \Omega_{m} h} E_{m}(t-h) .
$$

Выполняя аналогичные преобразования системы (3), приведем ее к следующему частному случаю (21):

$$
\left\{\begin{array}{l}
\frac{d \tilde{E}_{m}}{d s}=i \omega \tilde{E}_{m}+\varepsilon v(1+i \alpha)\left[\gamma_{m}\left(Z_{0}-Z_{m}\right)-1\right] \tilde{E}_{m}+\varepsilon \gamma e^{-i \Omega_{m} h+i \omega h \varepsilon^{-1}} \tilde{E}_{m}\left(s-h \varepsilon^{-1}\right), \\
\frac{d Z_{m}}{d s}=-Z_{m} \sum_{j=1}^{n} \gamma_{j}\left|\tilde{E}_{j}\right|^{2}+\gamma_{m}\left|\tilde{E}_{m}\right|^{2} Z_{0} / 2-\varepsilon(1+d) Z_{m} \\
\frac{d Z_{0}}{d s}=1+\sum_{j=1}^{n}\left|\tilde{E}_{j}\right|^{2}\left(Z_{j}-Z_{0}\right)-\varepsilon Z_{m} .
\end{array}\right.
$$

Семейство периодических решений системы нулевого приближения имеет вид

$$
E_{m}=c_{m} \exp (i \omega s), \quad Z_{m}=p_{m} Z_{0}, \quad Z_{0}=\left(\sum_{j=1}^{n}\left(1-p_{j}\right) c_{j}^{2}\right)^{-1},
$$

где $c_{m}$ - произвольные постоянные, $p_{m}$ обозначена следующая величина:

$$
p_{m}=\gamma_{m} c_{m}^{2}\left[2 \sum_{j=1}^{n} \gamma_{j} c_{j}^{2}\right]^{-1}=\gamma_{m}\left|E_{m}\right|^{2}\left[2 \sum_{j=1}^{n} \gamma_{j}\left|E_{j}\right|^{2}\right]^{-1} .
$$

Отметим, что $0 \leqslant p_{m} \leqslant 1 / 2$, если не все $c_{j}=0$ одновременно. При $n=2$ семейство периодических решений системы нулевого приближения можно записать в виде следующего вектора с вещественными компонентами:

$$
V_{0}(s)=\left(c_{1} \cos (\omega s), c_{1} \sin (\omega s), Z_{1}, c_{2} \cos (\omega s), c_{2} \sin (\omega s), Z_{2}, Z_{0}\right)^{T},
$$

где $Z_{0}, Z_{1}, Z_{2}$ определяются из $(42),(43)$ при $n=2$. В случае замены в $(41)$ величины $\tilde{E}_{m}=$ $\tilde{R}_{m} \exp \left(i \tilde{\theta}_{m}\right)$ это семейство решений представимо в виде

$$
V_{0}(s)=\left(c_{1}, \omega s, Z_{1}, c_{2}, \omega s, Z_{2}, Z_{0}\right)^{T} .
$$

Стандартная линеаризация $x^{\prime}=F(x)$ на $V_{0}(s)$ в соответствии с изложенной методикой приводит к системе $(24)$ с постоянной матрицей

$$
A=\left(\begin{array}{ccccccc}
0 & 0 & 0 & 0 & 0 & 0 & 0 \\
0 & 0 & 0 & 0 & 0 & 0 & 0 \\
-\gamma_{1} c_{1}\left[Z_{0}-2 Z_{1}\right] & 0 & -\gamma_{1} c_{1}^{2}-\gamma_{2} c_{2}^{2} & -\gamma_{2} c_{2} Z_{1} & 0 & 0 & \gamma_{1} c_{1}^{2} / 2 \\
0 & 0 & 0 & 0 & 0 & 0 & 0 \\
0 & 0 & 0 & 0 & 0 & 0 & 0 \\
-\gamma_{1} c_{1} Z_{2} & 0 & 0 & -\gamma_{2} c_{2}\left[Z_{0}-2 Z_{2}\right] & 0 & -\gamma_{1} c_{1}^{2}-\gamma_{2} c_{2}^{2} & \gamma_{2} c_{2}^{2} / 2 \\
2 c_{1}\left[Z_{1}-Z_{0}\right] & 0 & c_{1}^{2} & 2 c_{2}\left[Z_{2}-Z_{0}\right] & 0 & c_{2}^{2} & -c_{1}^{2}-c_{2}^{2}
\end{array}\right) .
$$


Среди собственных значений этой матрицы очевидно есть четыре нулевых. Еще три собственных значения матрицы $A$ являются собственными значениями подматрицы

$$
B_{2}=\left(\begin{array}{ccc}
-\gamma_{1} c_{1}^{2}-\gamma_{2} c_{2}^{2} & 0 & \gamma_{1} c_{1}^{2} / 2 \\
0 & -\gamma_{1} c_{1}^{2}-\gamma_{2} c_{2}^{2} & \gamma_{2} c_{2}^{2} / 2 \\
c_{1}^{2} & c_{2}^{2} & -c_{1}^{2}-c_{2}^{2}
\end{array}\right)=\left(\begin{array}{ccc}
-\sigma_{2} & 0 & \gamma_{1} c_{1}^{2} / 2 \\
0 & -\sigma_{2} & \gamma_{2} c_{2}^{2} / 2 \\
c_{1}^{2} & c_{2}^{2} & -\rho_{2}
\end{array}\right)
$$

Непосредственные вычисления показывают, что все $\lambda\left(B_{2}\right)$ вещественные отрицательные. Линейно независимые периодические решения сопряженной системы удобно выбрать следующим образом: $H_{0}(s)=(1,0,0,0,0,0,0)^{T}, H_{1}(s)=(0,1,0,0,0,0,0)^{T}, H_{2}(s)=(0,0,0,1,0,0,0)^{T}$, $H_{3}(s)=(0,0,0,0,1,0,0)^{T}$. Из условий $(26)$, переобозначая $\omega \varphi_{j} \rightarrow \varphi_{j}$, получим следующую систему интегро-дифференциальных уравнений, которую мы будем называть квазинормальной формой модели (3) в случае $Q \gg 1$ :

$$
\left\{\begin{array}{l}
c_{1}^{\prime}(t)=v\left(\frac{\gamma_{1}\left(1-p_{1}\right)}{\left(1-p_{1}\right) c_{1}^{2}(t)+\left(1-p_{2}\right) c_{2}^{2}(t)}-1\right) c_{1}(t)+\gamma \cos \left(\Omega_{1} h+\int_{-h}^{0} \varphi_{1}(t+r) d r\right) c_{1}(t-h), \\
\varphi_{1}(t)=v \alpha\left(\frac{\gamma_{1}\left(1-p_{1}\right)}{\left(1-p_{1}\right) c_{1}^{2}(t)+\left(1-p_{2}\right) c_{2}^{2}(t)}-1\right)-\gamma \sin \left(\Omega_{1} h+\int_{-h}^{0} \varphi_{1}(t+r) d r\right) \frac{c_{1}(t-h)}{c_{1}(t)} \\
c_{2}^{\prime}(t)=v\left(\frac{\gamma_{2}\left(1-p_{2}\right)}{\left(1-p_{1}\right) c_{1}^{2}(t)+\left(1-p_{2}\right) c_{2}^{2}(t)}-1\right) c_{2}(t)+\gamma \cos \left(\Omega_{2} h+\int_{-h}^{0} \varphi_{2}(t+r) d r\right) c_{2}(t-h), \\
\varphi_{2}(t)=v \alpha\left(\frac{\gamma_{2}\left(1-p_{2}\right)}{\left(1-p_{1}\right) c_{1}^{2}(t)+\left(1-p_{2}\right) c_{2}^{2}(t)}-1\right)-\gamma \sin \left(\Omega_{2} h+\int_{-h}^{0} \varphi_{2}(t+r) d r\right) \frac{c_{2}(t-h)}{c_{2}(t)} .
\end{array}\right.
$$

Величины $p_{m}$ определяются соотношением (43). Заметим, что система (44) заменами

$$
\varphi_{j}(t)=\theta_{j}^{\prime}(t), \quad c_{j}(t) e^{i \theta_{j}(t)}=E_{j}(t), \quad j=1,2,
$$

может быть преобразована к системе из двух комплексных уравнений

$$
\left\{\begin{array}{l}
\frac{d E_{1}}{d t}=v(1+i \alpha)\left[\frac{\gamma_{1}\left(1-p_{1}\right)}{\left(1-p_{1}\right)\left|E_{1}(t)\right|^{2}+\left(1-p_{2}\right)\left|E_{2}(t)\right|^{2}}-1\right] E_{1}+\gamma e^{-i \Omega_{1} h} E_{1}(t-h), \\
\frac{d E_{2}}{d t}=v(1+i \alpha)\left[\frac{\gamma_{2}\left(1-p_{2}\right)}{\left(1-p_{1}\right)\left|E_{1}(t)\right|^{2}+\left(1-p_{2}\right)\left|E_{2}(t)\right|^{2}}-1\right] E_{2}+\gamma e^{-i \Omega_{2} h} E_{2}(t-h) .
\end{array}\right.
$$

В отличие от случая $n=1$ системы (39) и (45) не сводятся одна к другой, величины $p_{m}$ в $(45)$ сами зависят от $\left|E_{m}\right|$ согласно (43). В общем случае при произвольном натуральном $n$ вектор $V_{0}(s)$ состоит из $1+3 n$ вещественных компонент, которые удобно представить как $n$ блоков из троек $c_{m}, \omega s, Z_{m}$, дополненных величиной $Z_{0}$, где $Z_{m}$ и $Z_{0}$ определяются из (42), (43). Подматрица, полученная из матрицы $A$ вычеркиванием последней строки и последнего столбца, тогда состоит из $n^{2}$ блоков размера $3 \times 3$, где главная диагональ занята подматрицами с двумя ненулевыми элементами, а остальные блоки имеют лишь один ненулевой элемент:

$$
\left(\begin{array}{ccc}
0 & 0 & 0 \\
0 & 0 & 0 \\
-\gamma_{m} c_{m}\left[Z_{0}-2 Z_{m}\right] & 0 & -\sigma_{n}
\end{array}\right), \quad\left(\begin{array}{ccc}
0 & 0 & 0 \\
0 & 0 & 0 \\
-\gamma_{i} c_{i} Z_{j} & 0 & 0
\end{array}\right)
$$

где использованы обозначения

$$
\sigma_{n}=\sum_{j=1}^{n} \gamma_{j} c_{j}^{2}, \quad \rho_{n}=\sum_{j=1}^{n} c_{j}^{2}
$$


Среди собственных значений $A$ есть $2 n$ нулевых (по числу нулевых строк $A$ ). Еще $1+n$ собственных значений матрицы $A$ являются собственными значениями подматрицы $(n+1) \times(n+1)$

$$
B_{n}=\left(\begin{array}{ccccc}
-\sigma_{n} & 0 & \ldots & 0 & \gamma_{1} c_{1}^{2} / 2 \\
0 & -\sigma_{n} & \ldots & 0 & \gamma_{2} c_{2}^{2} / 2 \\
\vdots & \vdots & \ddots & \vdots & \vdots \\
0 & 0 & \ldots & -\sigma_{n} & \gamma_{n} c_{n}^{2} / 2 \\
c_{1}^{2} & c_{2}^{2} & \ldots & c_{n}^{2} & -\rho_{n}
\end{array}\right)
$$

которая является внедиагонально неотрицательной матрицей Гурвица согласно [10] или относится к классу М-матриц согласно терминологии [11], т.е. удовлетворяет критерию СевастьяноваКотелянского (см. $[9,12]) ;$ следовательно, $\operatorname{Re} \lambda\left(B_{n}\right)<0$ для любого натурального $n$. Нулевым собственным значениям матрицы $A$ соответствуют единичные мультипликаторы системы $(24)$, число которых определяет число условий (26). Окончательный результат по аналогии со случаем $n=2$ можно сформулировать в форме обобщения системы (45) вида

$$
\frac{d E_{m}}{d t}=v(1+i \alpha)\left[\frac{\gamma_{m}\left(1-p_{m}\right)}{\sum_{j=1}^{n}\left(1-p_{j}\right)\left|E_{j}(t)\right|^{2}}-1\right] E_{m}+\gamma e^{-i \Omega_{m} h} E_{m}(t-h) .
$$

Численный анализ построенных квазинормальных форм для многомодовых моделей (2), (3) выполнялся в случае $n=2$. Рассматривались системы (38) при условии $\gamma_{1}=\gamma_{2}=\gamma$ и (44) при условии $\gamma_{1}=\gamma_{2}=1$. Значения остальных параметров полагались $v=100, \alpha=5, \Omega_{1} h=1$, $\Omega_{2} h=1,01$. Вычисления производились лишь для некоторых значений $h$ из диапазона, представленного на рис. 3. При изменении параметра $\gamma$ наблюдались такие же качественные изменения режимов от состояний равновесия до хаотических колебаний.

7. Результаты для систем с фильтром. Выполним в одномодовой модели (4) следующее преобразование переменных: $t=\varepsilon s, E=e^{-i \omega s} \tilde{E} / \sqrt{\varepsilon}, f=e^{-i \omega s} \tilde{f} / \sqrt{\varepsilon}$. В результате получим систему, которая является частным случаем $(21)$ :

$$
\left\{\begin{array}{l}
\frac{d \tilde{E}}{d s}=i \omega \tilde{E}+\varepsilon v(1+i \alpha) \tilde{E} Z-\varepsilon \gamma \tilde{f} \\
\frac{d \tilde{f}}{d s}=i \omega \tilde{f}+\varepsilon i \Delta \tilde{f}+\varepsilon \Lambda\left[e^{-\omega_{0} h+\omega h \varepsilon^{-1}} \tilde{E}\left(s-h \varepsilon^{-1}\right)-\tilde{f}\right] \\
\frac{d Z}{d s}=1-(1+Z)|\tilde{E}|^{2}-\varepsilon Z .
\end{array}\right.
$$

Решение системы «нулевого приближения» можно записать следующим образом:

$$
V_{0}(s)=\left(c \exp (i \omega s), \hat{c} \exp (i \omega s), c^{-2}-1\right)^{T} .
$$

В такой параметризации дальнейшие действия излагались в [3]. Запишем решение системы «нулевого приближения» в более удобной для вычислений форме, которая получается при полярной замене величин $E$ и $f$ :

$$
V_{0}(s)=\left(c, \omega s, \hat{c}, \omega s, c^{-2}-1\right)^{T} .
$$

Стандартная линеаризация $x^{\prime}=F(x)$ на $V_{0}(s)$ в соответствии с изложенной методикой приводит к системе $(24)$ с постоянной матрицей

$$
A=F^{\prime}\left(V_{0}(s)\right)=\left(\begin{array}{ccccc}
0 & 0 & 0 & 0 & 0 \\
0 & 0 & 0 & 0 & 0 \\
0 & 0 & 0 & 0 & 0 \\
0 & 0 & 0 & 0 & 0 \\
-2 c^{-1} & 0 & 0 & 0 & -c^{2}
\end{array}\right)
$$


Среди собственных значений этой матрицы очевидно есть четыре нулевых (и вещественное отрицательное $\left.-c^{2}\right)$, которым соответствуют линейно независимые решения $K_{i}(s), i=0, \ldots, 4$, записанные по столбцам матричной экспоненты:

$$
\exp (A s)=\left(\begin{array}{ccccc}
1 & 0 & 0 & 0 & 0 \\
0 & 1 & 0 & 0 & 0 \\
0 & 0 & 1 & 0 & 0 \\
0 & 0 & 0 & 1 & 0 \\
-2 c^{-3}\left(1-e^{-c^{2} s}\right) & 0 & 0 & 0 & e^{-c^{2} s}
\end{array}\right) .
$$

Линейно независимые периодические решения сопряженной системы удобно выбрать следующим образом: $H_{0}(s)=(1,0,0,0,0)^{T}, H_{1}(s)=(0,1,0,0,0)^{T}, H_{2}(s)=(0,0,1,0,0)^{T}, H_{3}(s)=(0,0,0,1,0)^{T}$. В данном случае замена (22), (23) несколько модифицируется:

$$
\begin{gathered}
V(s, \varepsilon)=V_{0}(\tau, \hat{\tau})+\varepsilon V_{1}(t, \tau, \hat{\tau})+\varepsilon^{2} V_{2}(t, \tau, \hat{\tau})+\ldots, \\
\frac{d \tau}{d s}=1+\varepsilon \varphi(t)+\varepsilon^{2} \psi(t)+\ldots, \quad \frac{d \hat{\tau}}{d s}=1+\varepsilon \hat{\varphi}(t)+\varepsilon^{2} \hat{\psi}(t)+\ldots
\end{gathered}
$$

Здесь $V_{0}(\tau, \hat{\tau})=(c \exp (i \omega \tau), \hat{c} \exp (i \omega \hat{\tau}), Z)^{T}$. Кроме того,

$$
\hat{\tau}(s)-\tau(s)=\varepsilon \int_{s_{0}}^{s}[\hat{\varphi}(\varepsilon r)-\varphi(\varepsilon r)+\ldots] d r=\int_{t_{0}}^{t}[\hat{\varphi}(r)-\varphi(r)] d r+O(\varepsilon) .
$$

В соответствии с изложенной методикой, собирая коэффициенты при первой степени $\varepsilon$, придем к следующей системе уравнений:

$$
\begin{aligned}
c^{\prime}(t) \frac{d V_{0}}{d c}+\hat{c}^{\prime}(t) \frac{d V_{0}}{d \hat{c}}+\varphi(t) \frac{d V_{0}}{d \tau}+\hat{\varphi}(t) & \frac{d V_{0}}{d \hat{\tau}}+\frac{d V_{1}}{d \tau}+\frac{d V_{1}}{d \hat{\tau}}= \\
& =A V_{1}+\Phi\left(V_{0}(\tau(s), \hat{\tau}(s)), V_{0}\left(\tau\left(s-h \varepsilon^{-1}\right), \hat{\tau}\left(s-h \varepsilon^{-1}\right)\right)\right) .
\end{aligned}
$$

Условие существования периодического (по $\tau, \hat{\tau}$ ) решения (48) состоит в ортогональности его неоднородной части функциям $H_{0}, H_{1}, H_{2}, H_{3}$. Отметим, что, сделав замену $\omega \varphi \rightarrow \varphi, \omega \hat{\varphi} \rightarrow \hat{\varphi}$, можно избавиться от $\omega$. Следующая система интегро-дифференциальных уравнений выступает в роли нормальной формы в задаче о локальной динамике уравнений (4) в окрестности решения $V_{0}$ невозмущенной предельной системы при $Q \rightarrow \infty$ :

$$
\begin{aligned}
& c^{\prime}(t)=v\left(\frac{1}{c(t)}-c(t)\right)+\gamma \cos \left(\int_{t_{0}}^{t}(\hat{\varphi}(r)-\varphi(r)) d r\right) \hat{c}(t), \\
& \varphi(t)=v \alpha\left(\frac{1}{c^{2}(t)}-1\right)+\gamma \sin \left(\int_{t_{0}}^{t}(\hat{\varphi}(r)-\varphi(r)) d r\right) \frac{\hat{c}(t)}{c(t)}, \\
& \hat{c}^{\prime}(t)=\Lambda\left[c(t-h) \cos \left(\omega_{0} h+\int_{-h}^{0} \varphi(t+r) d r+\int_{t_{0}}^{t}(\hat{\varphi}(r)-\varphi(r)) d r\right)-\hat{c}(t)\right], \\
& \hat{\varphi}(t)=\Delta-\Lambda \sin \left(\omega_{0} h+\int_{-h}^{t} \varphi(t+r) d r+\int_{t_{0}}^{t}(\hat{\varphi}(r)-\varphi(r)) d r\right) \frac{c(t-h)}{\hat{c}(t)} .
\end{aligned}
$$

Заметим, что квазинормальная форма (49) при выполнении замен $E(t)=c(t) e^{i \theta(t)}, \theta^{\prime}(t)=\varphi(t)$, $f(t)=\hat{c}(t) e^{i \hat{\theta}(t)}, \hat{\theta}^{\prime}(t)=\hat{\varphi}(t)$ допускает более компактную запись в терминах исходной системы (4):

$$
\left\{\begin{array}{l}
\frac{d E}{d t}=v(1+i \alpha)\left(|E|^{-2}-1\right) E+\gamma f \\
\frac{d f}{d t}=i \Delta f+\Lambda\left[E(t-h) e^{-i \omega_{0} h}-f\right]
\end{array}\right.
$$


Численный анализ показывает, что динамика (49) оказывается проще, чем (28). В пределах точности вычислений при $\gamma<v$ и $0<h<10$ вне зависимости от значений других параметров удавалось обнаружить лишь один аттрактор - устойчивое состояние равновесия, которому соответствует простой периодический режим в системе (4). Аналогичный результат получается и для модели Корюкина с оптическим фильтром в одномодовом случае. Именно, выполняя такое же преобразование переменных как в предыдущем случае, получим систему, которая является частным случаем (21):

$$
\left\{\begin{array}{l}
\frac{d \tilde{E}}{d s}=i \omega \tilde{E}+\varepsilon v(1+i \alpha)\left[\gamma_{1}\left(Z_{0}-Z\right)-1\right] \tilde{E}-\varepsilon \gamma \tilde{f} \\
\frac{d \tilde{f}}{d s}=i \omega \tilde{f}+\varepsilon i \Delta \tilde{f}+\varepsilon \Lambda\left[e^{-\omega_{0} h+\omega h \varepsilon^{-1}} \tilde{E}\left(s-h \varepsilon^{-1}\right)-\tilde{f}\right] \\
\frac{d Z}{d s}=\gamma_{1}|\tilde{E}|^{2} Z_{0} / 2-\gamma_{1}|\tilde{E}|^{2} Z-\varepsilon(1+d) Z \\
\frac{d Z_{0}}{d s}=1+|\tilde{E}|^{2}\left(Z-Z_{0}\right)-\varepsilon Z_{0} .
\end{array}\right.
$$

Решение системы «нулевого приближения» можно записать следующим образом:

$$
V_{0}(s)=\left(c \exp (i \omega s), \hat{c} \exp (i \omega s), c^{-2}, 2 c^{-2}\right)^{T},
$$

или в более удобной для вычислений форме, которая получается при полярной замене $E$ и $f$ :

$$
V_{0}(s)=\left(c, \omega s, \hat{c}, \omega s, c^{-2}, 2 c^{-2}\right)^{T} .
$$

Стандартная линеаризация $x^{\prime}=F(x)$ на $V_{0}(s)$ в соответствии с изложенной методикой приводит к системе $(24)$ с постоянной матрицей

$$
A=F^{\prime}\left(V_{0}(s)\right)=\left(\begin{array}{cccccc}
0 & 0 & 0 & 0 & 0 & 0 \\
0 & 0 & 0 & 0 & 0 & 0 \\
0 & 0 & 0 & 0 & 0 & 0 \\
0 & 0 & 0 & 0 & 0 & 0 \\
0 & 0 & 0 & 0 & -\gamma_{1} c^{2} & \gamma_{1} c^{2} / 2 \\
-2 c^{-1} & 0 & 0 & 0 & c^{2} & -c^{2}
\end{array}\right) .
$$

Среди собственных значений этой матрицы очевидно есть четыре нулевых, которым соответствуют следующие линейно независимые решения:

$$
\begin{array}{ll}
K_{0}(s)=\left(1,0,0,0,-2 c^{-3},-4 c^{-3}\right)^{T}, & K_{1}(s)=(0,1,0,0,0,0)^{T}, \\
K_{2}(s)=(0,0,1,0,0,0)^{T}, & K_{3}(s)=(0,0,0,1,0,0)^{T} .
\end{array}
$$

Линейно независимые периодические решения сопряженной системы удобно выбрать следующим образом: $H_{0}(s)=(1,0,0,0,0)^{T}, H_{1}(s)=(0,1,0,0,0)^{T}, H_{2}(s)=(0,0,1,0,0)^{T}, H_{3}(s)=(0,0,0,1,0)^{T}$.

Далее точно так же приходим к системе уравнений (48), в которой требуется выполнение условий ортогональности ее неоднородной части функциям $H_{0}, H_{1}, H_{2}, H_{3}$.

Отметим, что, сделав замену $\omega \varphi \rightarrow \varphi, \omega \hat{\varphi} \rightarrow \hat{\varphi}$, а также

$$
E(t)=c(t) e^{i \theta(t)}, \quad \theta^{\prime}(t)=\varphi(t), \quad f(t)=\hat{c}(t) e^{i \hat{\theta}(t)}, \quad \hat{\theta}^{\prime}(t)=\hat{\varphi}(t)
$$

получаем компактную запись итоговой системы:

$$
\left\{\begin{array}{l}
\frac{d E}{d t}=v(1+i \alpha)\left(\gamma_{1}|E|^{-2}-1\right) E+\gamma f \\
\frac{d f}{d t}=i \Delta f+\Lambda\left[E(t-h) e^{-i \Omega h}-f\right] .
\end{array}\right.
$$

Нормировочной заменой $\gamma_{1}^{-1 / 2} E \rightarrow E$ и переобозначением $\Omega \rightarrow \omega_{0}$ она приводится к виду (50).

Таким образом, динамика обеих моделей (5) и (6) при $n=1$ в случае $Q \gg 1$ математически описывается одной и той же системой, и в этом смысле они не отличаются друг от друга. 
Другая особенность рассмотренных выше построений заключается в том, что полученные здесь матрицы $A$ отличаются от таковых в случае одномодовых моделей без фильтра только наличием дополнительных нулевых элементов. На выходе это дает дополнительные уравнения, описывающие воздействие оптического фильтра.

Такие же особенности имеют место в случае многомодовых моделей. Опуская громоздкие преобразования, аналогичные преобразованиям в случае многомодовых моделей без фильтра, выпишем окончательные уравнения.

В случае модели (5) при $n=2$ приходим к следующей системе интегро-дифференциальных уравнений:

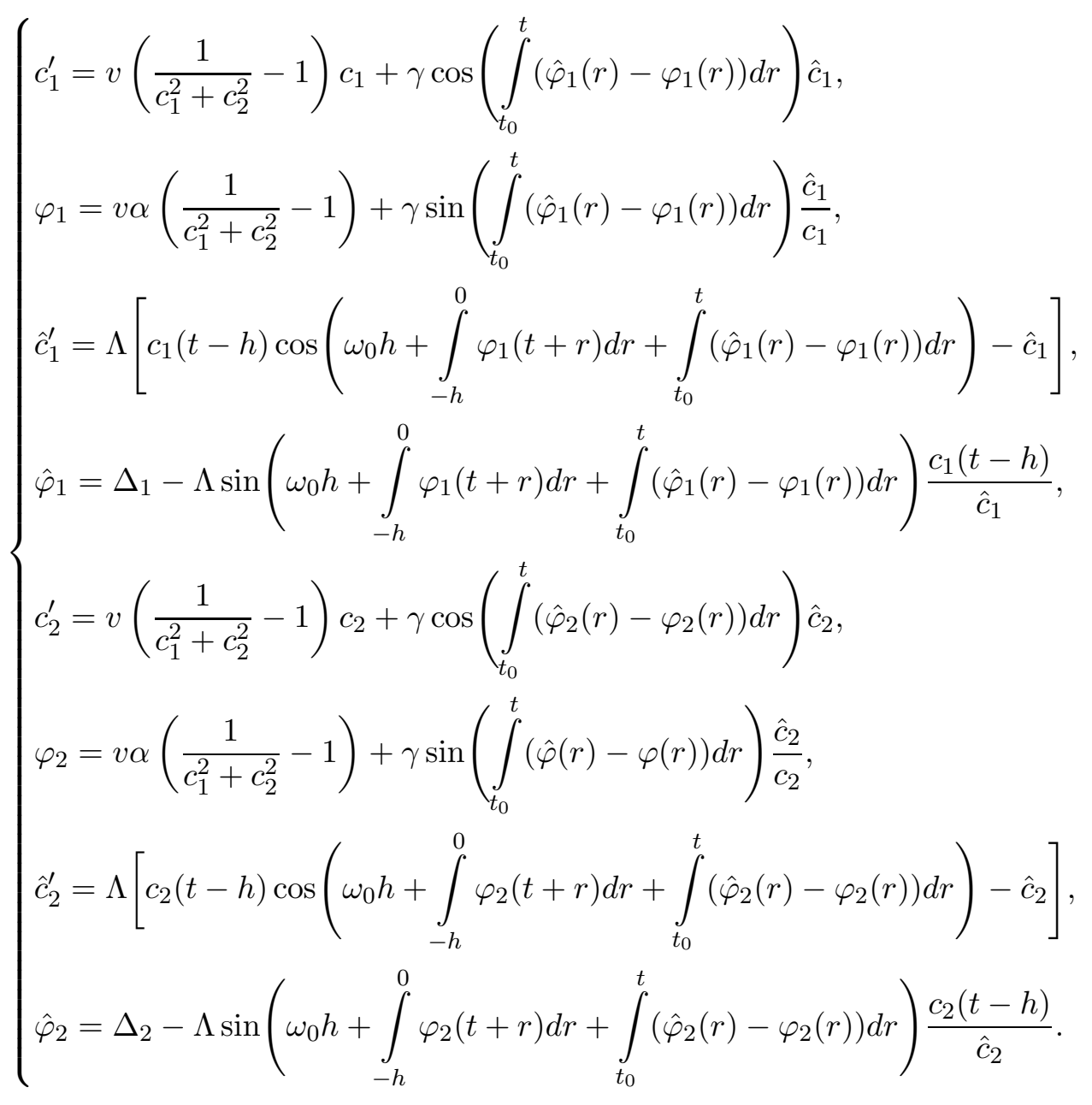

Выполняя замены

$$
E_{m}(t)=c_{m}(t) e^{i \theta_{m}(t)}, \quad \theta_{m}^{\prime}(t)=\varphi_{m}(t), \quad f_{m}(t)=\hat{c}_{m}(t) e^{i \hat{\theta}_{m}(t)}, \quad \hat{\theta}_{m}^{\prime}(t)=\hat{\varphi}_{m}(t),
$$

получаем компактную запись итоговой системы:

$$
\left\{\begin{array}{l}
\frac{d E_{1}}{d t}=v(1+i \alpha)\left(\frac{1}{\left|E_{1}\right|^{2}+\left|E_{2}\right|^{2}}-1\right) E_{1}+\gamma f_{1}, \\
\frac{d f_{1}}{d t}=i \Delta_{1} f_{1}+\Lambda\left[E_{1}(t-h) e^{-i \Omega_{1} h}-f_{1}\right], \\
\frac{d E_{2}}{d t}=v(1+i \alpha)\left(\frac{1}{\left|E_{1}\right|^{2}+\left|E_{2}\right|^{2}}-1\right) E_{2}+\gamma f_{2}, \\
\frac{d f_{2}}{d t}=i \Delta_{2} f_{2}+\Lambda\left[E_{2}(t-h) e^{-i \Omega_{2} h}-f_{2}\right] .
\end{array}\right.
$$


В случае модели (6) при $n=2$ получаем систему

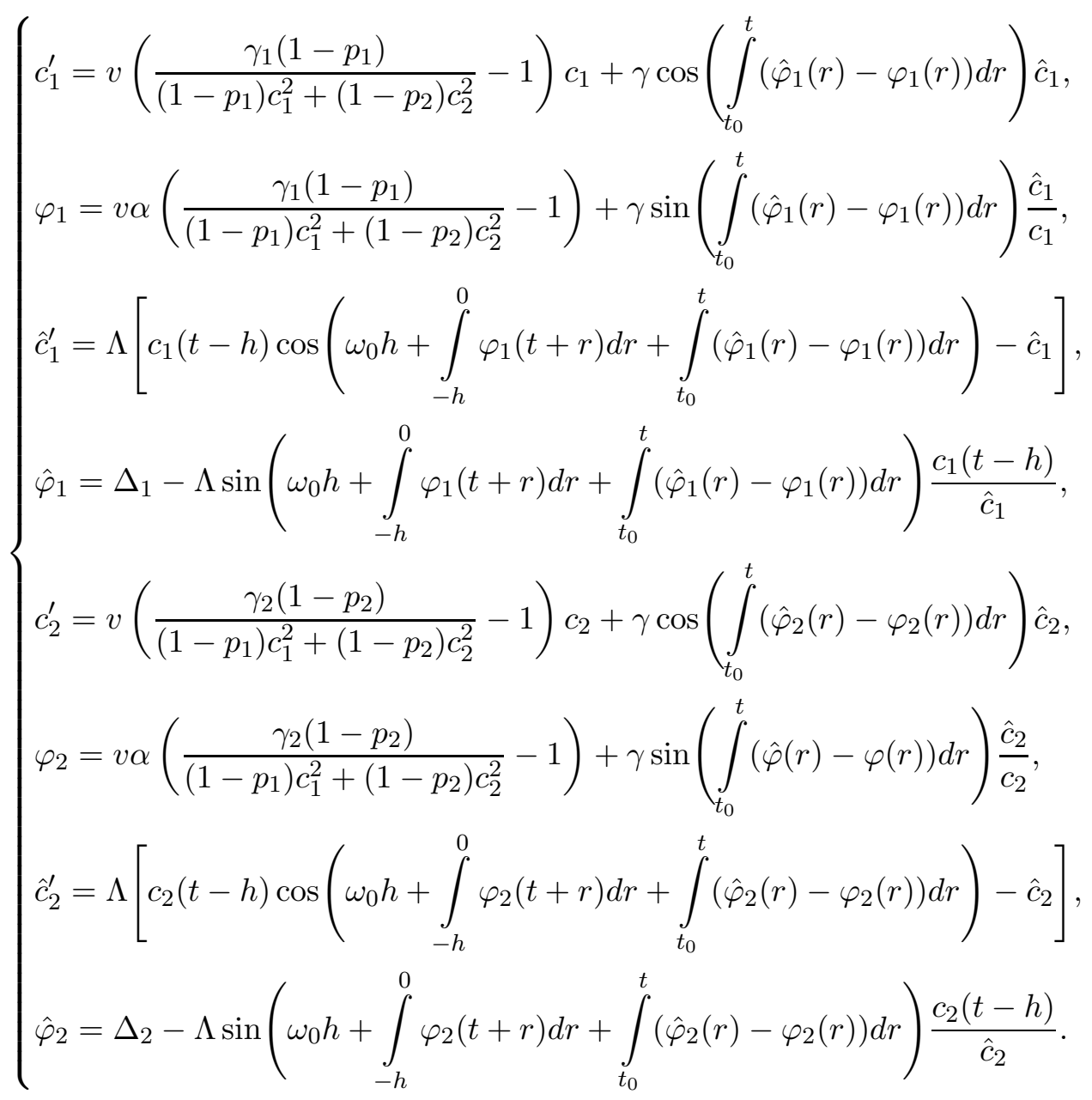

Заменами

$$
E_{m}(t)=c_{m}(t) e^{i \theta_{m}(t)}, \quad \theta_{m}^{\prime}(t)=\varphi_{m}(t), \quad f_{m}(t)=\hat{c}_{m}(t) e^{i \hat{\theta}_{m}(t)}, \quad \hat{\theta}_{m}^{\prime}(t)=\hat{\varphi}_{m}(t)
$$

она приводится к виду

$$
\left\{\begin{array}{l}
\frac{d E_{1}}{d t}=v(1+i \alpha)\left(\frac{\gamma_{1}\left(1-p_{1}\right)}{\left(1-p_{1}\right)\left|E_{1}\right|^{2}+\left(1-p_{2}\right)\left|E_{2}\right|^{2}}-1\right) E_{1}+\gamma f_{1} \\
\frac{d f_{1}}{d t}=i \Delta_{1} f_{1}+\Lambda\left[E_{1}(t-h) e^{-i \Omega_{1} h}-f_{1}\right] \\
\frac{d E_{2}}{d t}=v(1+i \alpha)\left(\frac{\gamma_{2}\left(1-p_{2}\right)}{\left(1-p_{1}\right)\left|E_{1}\right|^{2}+\left(1-p_{2}\right)\left|E_{2}\right|^{2}}-1\right) E_{2}+\gamma f_{2} \\
\frac{d f_{2}}{d t}=i \Delta_{2} f_{2}+\Lambda\left[E_{2}(t-h) e^{-i \Omega_{2} h}-f_{2}\right] .
\end{array}\right.
$$

Численный анализ построенных квазинормальных форм для многомодовых моделей (5), (6) выполнялся в случае $n=2$. Рассматривались системы (53) при условии $\gamma_{1}=\gamma_{2}=\gamma$ и (55) при условии $\gamma_{1}=\gamma_{2}=1$. Величины $\Delta_{1}, \Delta_{2}, \Lambda$, характеризующие фильтр, менялись в достаточно широких пределах: от $10^{-4}$ до $10^{4}$. Значения остальных параметров полагались $v=100, \alpha=5$, $\Omega_{1} h=1, \Omega_{2} h=1,01$.

В пределах точности вычислений при $0<\gamma<v$ и $0<h<10$ системах (53) и (55) вне зависимости от значений других параметров удавалось обнаружить лишь один тип аттрактора устойчивое состояние равновесия, которому соответствует простой периодический режим в системах (5) и (6). 
8. Нормализация в неавтономном случае. Полученные в разделах 4, 6 результаты распространяются на случай, когда параметры рассматриваемых систем являются гладкими ограниченными периодическими функциями времени $t$.

При этом, как отмечено, например, в [26], физически в модели Ланга-Кобаяси проще всего управлять параметрами $h$ и $Q$.

Согласно [3] наиболее содержательные результаты в случае системы (1) удалось получить при быстро осциллирующем параметре запаздывания:

$$
h=h_{0}+h_{1} \sin \left(t \varepsilon^{-2}\right), \quad 0<h_{1}<h_{0} .
$$

Именно, выполняя стандартные замены в уравнениях (1), усредняя их по «быстрому» времени $s \varepsilon^{-1}=t \varepsilon^{-2}$ и действуя далее в соответствии с изложенным алгоритмом нормализации, получим следующую систему с распределенным запаздыванием:

$$
\left\{\begin{array}{l}
c^{\prime}(t)=v\left(\frac{1}{c(t)}-c(t)\right)+\frac{1}{\pi} \int_{-1}^{1} \frac{\gamma}{\sqrt{1-r^{2}}} \cos \left(\omega_{0} h(r)+\int_{-h(r)}^{0} \varphi(t+u) d u\right) c(t-h(r)) d r, \\
\varphi(t)=\left(\frac{1}{c^{2}(t)}-1\right)-\frac{1}{\pi} \int_{-1}^{1} \frac{\gamma}{\sqrt{1-r^{2}}} \sin \left(\omega_{0} h(r)+\int_{-h(r)}^{0} \varphi(t+u) d u\right) \frac{c(t-h(r))}{c(t)} d r .
\end{array}\right.
$$

Здесь $h(r)=h_{0}+h_{1} r$. Эта же система получается в случае модели (3) при $n=1$.

Численный анализ системы (57) показывает, что уже при соотношении $h_{1} / h_{0}=0,01$ происходит ощутимый сдвиг вправо по параметру $\gamma$ всех границ между областями на бифуркационной диаграмме из раздела 5 (см. рис. 3). При $h_{1} / h_{0}=0,2$ в системе $(57)$ практически всюду в рассматриваемой области параметров устанавливается стационарный режим, которому соответствует простой периодический режим в усредненной системе (1).

В случае модели (2) при $n=2$, действуя аналогичным образом, приходим к следующей системе интегрально-дифференциальных уравнений:

$$
\left\{\begin{array}{l}
c_{1}^{\prime}=v\left(\frac{1}{c_{1}^{2}+c_{2}^{2}}-1\right) c_{1}+\frac{1}{\pi} \int_{-1}^{1} \frac{\gamma}{\sqrt{1-r^{2}}} \cos \left(\Omega_{1} h(r)+\int_{-h(r)}^{0} \varphi_{1}(t+u) d u\right) c_{1}(t-h(r)) d r, \\
\varphi_{1}=v \alpha\left(\frac{1}{c_{1}^{2}+c_{2}^{2}}-1\right)-\frac{1}{\pi} \int_{-1}^{1} \frac{\gamma}{\sqrt{1-r^{2}}} \sin \left(\Omega_{1} h(r)+\int_{-h(r)}^{0} \varphi_{1}(t+u) d u\right) \frac{c_{1}(t-h(r))}{c_{1}(t)} d r, \\
c_{2}^{\prime}=v\left(\frac{1}{c_{1}^{2}+c_{2}^{2}}-1\right) c_{2}+\frac{1}{\pi} \int_{-1}^{1} \frac{\gamma}{\sqrt{1-r^{2}}} \cos \left(\Omega_{2} h(r)+\int_{-h(r)}^{0} \varphi_{2}(t+u) d u\right) c_{2}(t-h(r)) d r, \\
\varphi_{2}=v \alpha\left(\frac{1}{c_{1}^{2}+c_{2}^{2}}-1\right)-\frac{1}{\pi} \int_{-1}^{1} \frac{\gamma}{\sqrt{1-r^{2}}} \sin \left(\Omega_{2} h(r)+\int_{-h(r)}^{0} \varphi_{2}(t+u) d u\right) \frac{c_{2}(t-h(r))}{c_{2}(t)} d r .
\end{array}\right.
$$


В случае модели (3) при $n=2$ получаем систему

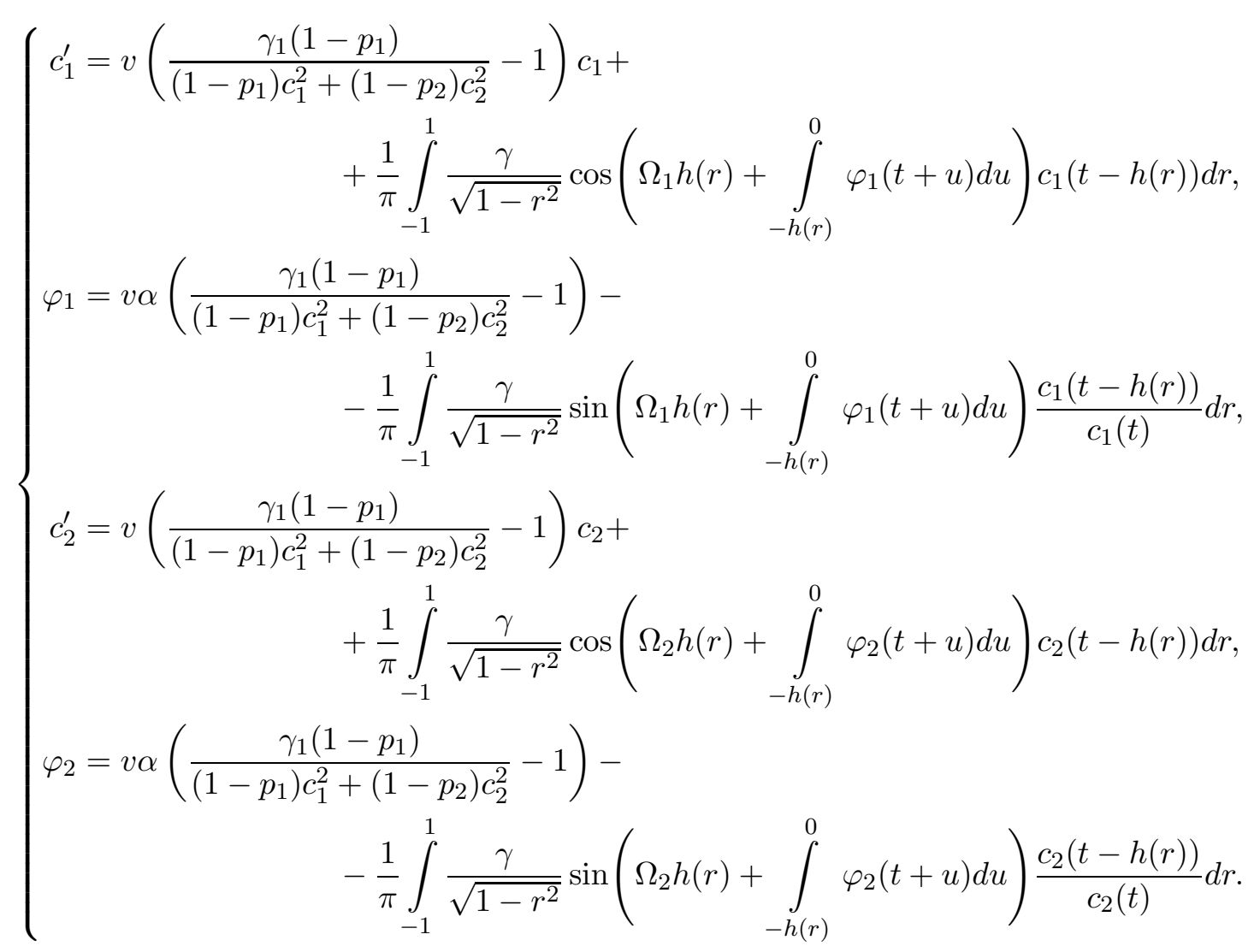

Численный анализ систем (58) при условии $\gamma_{1}=\gamma_{2}=\gamma$ и (59) при условии $\gamma_{1}=\gamma_{2}=1$ выполнялся при $v=100, \alpha=5, \Omega_{1} h=1, \Omega_{2} h=1,01$. Здесь, как и в одномодовом случае, уже при $h_{1} / h_{0}=0,01$ происходит существенное увеличение областей устойчивости более простых режимов за счет областей с более сложной динамикой. При $h_{1} / h_{0}=0,25$ в системах (58) и (59) практически всюду в рассматриваемой области параметров устанавливаются стационарные режимы, которым соответствуют простые периодический режимы в усредненных системах (2) и (3) соответственно.

9. Заключение. Выполнено математическое исследование нескольких моделей полупроводникового лазера с запаздывающей обратной связью. Построены новые эволюционные уравнения, так называемые квазинормальные формы, которые описывают динамику исходных систем в окрестности предельных семейств периодических решений при асимптотически больших значениях параметра накачки. Аттракторы изначальных модельных задач и нормализованных уравнений связаны между собой стандартным образом: устойчивому состоянию равновесия, циклу квазинормальной формы соответствуют устойчивый цикл, тор в исходной системе.

Основной особенностью использованного в работе подхода является исключение «быстрых» движений. В результате остаются только уравнения для «медленных» амплитуд, которые определяют «в главном» поведение решений исходных систем. При этом редуцированные системы уже не содержат параметр, который рассматривается как асимптотически большой (малый), что серьезно облегчает численный счет.

В случае большой величины накачки модели с фильтром, несмотря на кажущуюся сложность, демонстрируют более простую динамику, нежели системы без фильтра. Вне зависимости от интенсивности запаздывающей оптической обратной связи в системах (53) и (55) устанавливается простой периодический режим, соответствующий стабильному режиму генерации. Аналогичные выводы можно сделать, исследуя динамику систем (58) и (59) при быстро осциллирующем запаздывании. Соответственно фильтрование отраженного электромагнитного излучения и высокочастотная вибрация отражающих поверхностей представляют собой действенные механизмы преодоления явления детерминированного динамического хаоса. Отмеченный стабилизирующий 
эффект от использования оптического фильтра или быстро вибрирующих зеркал может быть принят во внимание в различных приложениях оптико-электронных систем.

\section{СПИСОК ЛИТЕРАТУРЫ}

1. Васильева А. Б., Бутузов В. Ф. Асимптотические разложения решений сингулярно возмущенных уравнений. - М.: Наука, 1973.

2. Глазков Д. В. Особенности динамики модели Ланга-Кобаяши в одном критическом случае// Модел. анал. информ. сист. - 2008. - 15, № 2. - С. 36-45.

3. Глазков Д. В. Качественный анализ сингулярно возмущенных моделей одного класса оптикоэлектронных систем// Изв. вузов. Прикл. нелин. динам. - 2008. - 16, № 4. - С. 167-181.

4. Глазков Д. В. Простейшие квазинормальные формы моделей многомодовых лазеров с запаздыванием// Вестн. ЯрГУ. Сер. естеств. техн. науки. - 2013. - 2. - С. 49-56.

5. Кащенко C. А. Бифуркации цикла в сингулярно возмущенных нелинейных автономных системах// Изв. РАЕН. Сер. мат. мат. модел. информ. управл. - 1998. - 2, № 4. - С. 5-53.

6. Кащенко С. А. Локальная динамика нелинейных сингулярно возмущенных систем с запаздыванием// Диффер. уравн. - 1999. - 35, № 10. - С. 1343-1355.

7. Кащенко С. А. Бифуркации в окрестности цикла при малых возмущениях с большим запаздыванием// Ж. вычисл. мат. мат. физ. - 2000. - 4. - С. 659-668.

8. Корюкин И. В. Динамика многомодового полупроводникового лазера с оптической обратной связью// Физ. техн. полупроводн. - 2009. - 43, № 3. - С. 405-411.

9. Котелянский Д. М. О некоторых свойствах матриц с положительными элементами// Мат. сб. - 1952. - 31, № 3. - С. 495-506.

10. Перов А. И. Новые признаки устойчивости линейных систем дифференциальных уравнений с постоянными коэффициентами// Изв. вузов. Мат. - 2014. - 9. - С. 49-58.

11. Перчев Н. В., Пичугин Б. Ю., Пичугина А. Н. Применение М-матриц для исследования математических моделей живых систем// Мат. биол. биоинформ. - 2018. - 13, № 1. - С. 208-237.

12. Севастьянов Б. А. Ветвящиеся процессы. - М.: Наука, 1971.

13. Ханин Я. И. Основы динамики лазеров. - М.: Наука, 1999.

14. Fischer I., Hess O., Elsässer W. and Göbel E. High-dimensional chaotic dynamics of an external cavity semiconductor laser// Phys. Rev. Lett. — 1994. — 73, № 16. — P. 2188-2191.

15. Grassberger P., Procaccia I. Estimation of the Kolmogorov entropy from a chaotic signal// Phys. Rev. A. - 1983. - 28, № 4. - P. 2591-2593.

16. Grassberger P., Procaccia I. Measuring the strangeness of strange attractors// Phys. D. - 1983. - 9, № 1-2. - P. 189-208.

17. Green K., Krauskopf B. Mode structure of semiconductor laser subject to filtered optical feedback// Opt. Commun. - 2006. - 258. - P. 243-255.

18. Grigorieva E. V., Haken H., Kaschenko S. A. Theory of quasiperiodicity in model of lasers with delayed optoelectronic feedback// Opt. Commun. - 1999. - 165. - P. 279-292.

19. Grigorieva E. V. Quasiperiodicity in Lang-Kobayashi model of lasers with delayed optical feedback// Nonlin. Phenom. Compl. Systems. - 2001. - 4, № 4. - P. 333-340.

20. Heil T., Fischer I., Elsässer W., Krauskopf B., Green K., Gavrielides A. Delay dynamics of semiconductor lasers with short external cavities: Bifurcation scenarios and mechanisms// Phys. Rev. E. - 2003. - 67. - 066214 .

21. Kaschenko S. A. Normalization in the systems with small diffusion// Int. J. Bifurcat. Chaos. - 1996. 6, № 7 . - P. 1093-1109.

22. Kaschenko S. A. Bifurcational features in systems of nonlinear parabolic equations with weak diffusion// Int. J. Bifurcat. Chaos. - 2005. - 15, № 11. - P. 3595-3606.

23. Lang R., Kobayashi K. External optical feedback effects on semiconductor injection laser properties// IEEE J. Quant. Electron. - 1980. - 16, № 1. - P. 347-355.

24. Levine A. M., Tartwijk G. H. M., Lenstra D. and Erneux T. Diode lasers with optical feedback: Stability of the maximum gain mode// Phys. Rev. A. - 1995. - 52, № 5. - P. 3436-3439.

25. Lythe G., Erneux T., Gavrielides A. and Kovanis V. Low pump limit of the bifurcation to periodic intensities in a semiconductor laser subject to external optical feedback// Phys. Rev. A. - 1997. — 55, № 6. P. 4443-4448. 
26. Mendez J. M., Laje R., Giudici M., Aliaga J. and Mindlin G. B. Dynamics of periodically forced semiconductor laser with optical feedback// Phys. Rev. E. - 2001. - 63. - 066218.

27. Ohtsubo J. Semiconductor Lasers. Stability, Instability and Chaos. — Springer, 2017.

28. Rottschafer V., Krauskopf B. A three-parameter study of external cavity modes in semiconductor lasers with optical feedback. - Proc. IFAC-TDS, 2004.

29. Sacher J., Elsässer W., Göbel E. O. Intermittency in the coherence collapse of a semiconductor laser with external feedback// Phys. Rev. Lett. — 1989. - 63, № 20. - P. 2224-2227.

30. Sano T. Antimode dynamics and chaotic itinerancy in the coherence collapse of semiconductor lasers with optical feedback// Phys. Rev. A. - 1994. - 50, № 3. - P. 2719-2726.

31. Sciamanna M., Megret P., and Blondel M. Hopf bifurcation cascade in small- $\alpha$ laser diodes subject to optical feedback// Phys. Rev. E. - 2004. - 69. - 046209.

32. Soriano M. C., Garcia-Ojalvo J., Mirasso C. R., and Fischer I. Complex photonics: Dynamics and applications of delay-coupled semiconductors lasers// Rev. Mod. Phys. — 2013. — 85, № 1. — P. 421-470.

33. Tabaka A., Panajotov K., Veretennicoff I., Sciamanna M. Bifurcation study of regular pulse packages in laser diodes subject to optical feedback// Phys. Rev. E. — 2004. — 70. — 036211.

34. Van Tartwijk G. H. M. and Agrawal G. P. Laser instabilities: a modern perspective// Progr. Quant. Electr. - 1998. - 22. - P. 43-122.

35. Vicktorov E. A., Mandel P. Low frequency fluctuations in a multimode semiconductor laser with optical feedback// Phys. Rev. Lett. — 2000. — 85, № 15. — P. 3157-3160.

36. Wolf A., Swift J. B., Swinney H. L., Vastano J. A. Determining Lyapunov exponents from a time series// Phys. D. - 1985. - 16, № 3. - P. 285-317.

37. Ye J., Li H., McInerny J. M. Period-doubling route to chaos in a semiconductor laser with weak optical feedback// Phys. Rev. A. - 1993. - 47, № 3. - P. 2249-2252.

Глазков Дмитрий Владимирович

Ярославский государственный университет им. П. Г. Демидова

E-mail: d.glazkov@uniyar.ac.ru 